\title{
Generalized connections, spinors, and integrability of generalized structures on Courant algebroids
}

\author{
Vicente Cortés and Liana David
}

January 20, 2021

\begin{abstract}
We present a characterization, in terms of torsion-free generalized connections, for the integrability of various generalized structures (generalized almost complex structures, generalized almost hypercomplex structures, generalized almost Hermitian structures and generalized almost hyper-Hermitian structures) defined on Courant algebroids. We develop a new, self-contained, approach for the theory of Dirac generating operators on regular Courant algebroids with scalar product of neutral signature. As an application we provide a criterion for the integrability of generalized almost Hermitian structures $(G, \mathcal{J})$ and generalized almost hyper-Hermitian structures $\left(G, \mathcal{J}_{1}, \mathcal{J}_{2}, \mathcal{J}_{3}\right)$ defined on a regular Courant algebroid $E$ with scalar product of neutral signature in terms of canonically defined differential operators on spinor bundles associated to $E_{ \pm}$(the subbundles of $E$ determined by the generalized metric $G$ ). ॥
\end{abstract}

2000 Mathematics Subject Classification: MSC 53D18 (primary); 53C15 (secondary). Keywords: Courant algebroids, generalized complex structures, generalized Kähler structures, generalized hypercomplex structures, generalized hyper-Kähler structures, generating Dirac operators. 


\section{Contents}

1 Introduction 3

I First Part: Generalized connections and integrability

2 Preliminary material 5

2.1 Courant algebroids . . . . . . . . . . . . . . 6

$2.2 \quad$ E-connections and generalized connections . . . . . . . . 7

2.3 Generalized metrics . . . . . . . . . . . . . 8

2.4 Generalized complex and hyper-complex structures . . . . . . 9

2.5 Generalized Kähler and hyper-Kähler structures . . . . . . . . 10

2.6 Intrinsic torsion of a generalized structure . . . . . . . . . . 11

2.7 Application to Born geometry . . . . . . . . . . . . . 13

3 Generalized almost complex structures $\quad \mathbf{1 5}$

3.1 Intrinsic torsion of a generalized almost complex structure . . 15

3.2 Integrability using torsion-free generalized connections . . . . 16

4 Generalized almost hypercomplex structures 19

4.1 Intrinsic torsion of a generalized almost hypercomplex structure 19

4.2 Integrability using torsion-free generalized connections . . . . 20

5 Generalized almost Hermitian structures: integrability and torsion-free generalized connections

6 Generalized almost hyper-Hermitian structures: integrability and torsion-free generalized connections

II Second Part: Dirac generating operators

7 The space of local Dirac generating operators

8 The canonical Dirac generating operator 33

9 Standard form of the canonical Dirac generating operator 38

III Third Part: Applications 
10 Generalized almost Hermitian structures: integrability and spinors

11 Appendix 49

$11.1 \mathbb{Z}_{2}$-graded algebras and Clifford algebras . . . . . . . . . 49

11.2 Integrability of generalized almost complex structures and spinors $\mathbf{5 0}$

\section{Introduction}

Generalized complex geometry is a well established field in present mathematics. It unifies complex and symplectic geometry and represents an important direction of current research in differential geometry and theoretical physics. In generalized geometry, the role of the tangent bundle $T M$ of a manifold $M$ is played by the generalized tangent bundle $\mathbb{T} M:=T M \oplus T^{*} M$, or, more generally, by a Courant algebroid. Many classical objects from differential geometry (including almost complex, almost Hermitian, almost hypercomplex and almost hyper-Hermitian structures) were defined and studied by several authors in this more general setting, see e.g. [16] (also [4, 5, 6]).

While the integrability of such generalized structures is defined and understood in terms of the Dorfmann bracket of the Courant algebroid, it seems that characterizations in terms of torsion-free generalized connections (analogous to the standard characterizations of integrability from classical geometry) are missing.

In the first part of the present paper we fill this gap by answering this natural question. We shall do this by a careful analysis of the space of generalized connections which are 'adapted', i.e. preserve, a given generalized structure $Q$ on a Courant algebroid $E$. In analogy with the classical case, we introduce the notion of intrinsic torsion $t_{Q}$ of $Q$, see Definition 15. This will play an important role in our treatment, when $Q$ is a generalized almost complex structure or a generalized almost hypercomplex structure. We compute the intrinsic torsion for these structures and we prove that their integrability is equivalent to the existence of a torsion-free adapted generalized connection, see Theorem 23 and Theorem 32. In the same framework, we prove that a generalized almost Hermitian structure on a Courant algebroid is integrable if and only if it admits a torsion-free adapted generalized connection, see Theorem 36. A similar integrability criterion for generalized almost hyperHermitian structures is proved in Theorem 38. Our treatment shows that in generalized geometry the torsion-free condition on a generalized connection $D$ adapted to a generalized structure $Q$ does in general no longer determine $D$ uniquely, even if the classical structure generalized by $Q$ has a unique 
torsion-free adapted connection. This was noticed already in [13, 10, for generalized Riemannian metrics: for a given generalized Riemannian metric $G$, there is an entire family of generalized connections which are torsion-free and preserve $G$. We will show that the same holds for the structures considered in this paper, for instance, for generalized hypercomplex structures: the Obata connection [22, 3] (see also [14]) of a hypercomplex structure is replaced in generalized geometry by an entire family of generalized connections.

In more general terms, the theory developed here allows to decide whether a given generalized structure $Q$ on a Courant algebroid admits an adapted generalized connection with prescribed torsion and to describe the space of all such generalized connections, see Proposition [17. A torsion-free generalized connection adapted to $Q$, for instance, exists if and only if the intrinsic torsion of $Q$ vanishes and is unique if and only if the generalized first prolongation $\mathfrak{h}^{\langle 1\rangle}$ of the Lie algebra $\mathfrak{h}$ of the structure group of $Q$ is zero. As a further application of the generalized first prolongation we present an alternative proof for the uniqueness of the canonical connection of a Born structure defined in [9] (see Section 2.7).

In the second part of the paper we present a self-contained treatment of the canonical Dirac generating operator on a regular Courant algebroid with scalar product of neutral signature. A Courant algebroid is regular if its anchor has constant rank. Regular Courant algebroids form an important class of Courant algebroids, which was studied systematically in [7]. A Dirac generating operator is a first order odd differential operator on a suitable irreducible spinor bundle which encodes the anchor and the Dorfman bracket of the Courant algebroid. We recover the crucial result of [1] (see also [15] and [8], [10]) namely that any regular Courant algebroid $E$ with scalar product of neutral signature admits a canonical Dirac generating operator $\not d$ and we express $\not d$ in terms of a dissection of $E$. Such an expression for the canonical Dirac generating operator allows a better understanding of the relation between $\phi$ and the structure of the regular Courant algebroid.

The third part of the paper is devoted to applications. We assume that $E$ is a regular Courant algebroid with scalar product of neutral signature. Owing to the failure of uniqueness of generalized connections adapted to various generalized structures $Q$ on $E$ it is natural to search for characterizations of the integrability of $Q$ using the canonical Dirac generating operator of $E$. This was done in [1] when $Q=\mathcal{J}$ is a generalized almost complex structure. Here we present a criterion for a generalized almost Hermitian structure $(G, \mathcal{J})$ on $E$ to be generalized Kähler in terms of two canonical Dirac operators and the pure spinors associated to $\left.\mathcal{J}\right|_{E_{ \pm}}$(where $E_{ \pm}$are the subbundles of $E$ determined by the generalized metric $G$ ), see Theorem 67. Our arguments use, besides the theory of Dirac generating operators, the 
results on generalized connections adapted to a generalized almost Hermitian structure, developed in the first part of the paper. A similar spinorial characterization for generalized hyper-Kähler structures on regular Courant algebroids with scalar product of neutral signature is obtained in Corollary 70.

In the appendix, intended for completeness of our exposition, we briefly recall basic facts we need on the theory of $\mathbb{Z}_{2}$-graded algebras and the integrability criterion in terms of pure spinors for generalized almost complex structures on regular Courant algebroids with scalar product of neutral signature, mentioned above.

In Parts II and III of the paper the assumption that the scalar product of the Courant algebroid $E$ has neutral signature plays a crucial role. Then the spinor bundles $S$ on which the Dirac generating operators act are irreducible $\mathbb{Z}_{2}$-graded $\mathrm{Cl}(E)$-bundles, with the essential property that any $\mathrm{Cl}(E)$-bundle morphism $f: S \rightarrow S$ is a multiple of the identity. (More generally, considering signatures $(t, s)$ with $t-s \equiv 0$ or $2(\bmod 8)$, would ensure the same property, where $t$ stands for the index and the Clifford relation is $v^{2}=\langle v, v\rangle$ 1.) It would be interesting to extend the theory of Dirac generating operators to Courant algebroids of other signatures.

\section{Part I}

\section{Preliminary material}

We start by reviewing the basic definitions we need on Courant algebroids, generalized connections, their torsion (see [10]) and the definition of the generalized structures we shall consider in this paper. We prove a basic property of the Nijenhuis tensor of a generalized almost complex structure (see Lemma 9), we introduce the notion of intrinsic torsion of a generalized structure $Q$ on a Courant algebroid and we describe the space of generalized connections adapted to $Q$, with prescribed torsion, as an affine space modeled on the space of sections of a vector bundle (see Proposition [17). The fibers of this vector bundle are isomorphic to the generalized first prolongation $\mathfrak{h}^{\langle 1\rangle}$ of the structure group $H$ of $Q$, a notion which will be defined for any Lie subgroup $H \subset \mathrm{O}(k, \ell)$. 


\subsection{Courant algebroids}

Definition 1. A Courant algebroid on a manifold $M$ is a vector bundle $E \rightarrow M$ equipped with a non-degenerate symmetric bilinear form $\langle\cdot, \cdot\rangle \in$ $\Gamma\left(\operatorname{Sym}^{2}\left(E^{*}\right)\right)$ (called the scalar product), a bilinear operation $[\cdot, \cdot]$ (called the Dorfman bracket) on the space of smooth sections $\Gamma(E)$ of $E$ and a homomorphism of vector bundles $\pi: E \rightarrow T M$ (called the anchor), such that the following conditions are satisfied: for all sections $u, v, w \in \Gamma(E)$,

C1) $[u,[v, w]]=[[u, v], w]+[v,[u, w]]$;

C2) $\pi([u, v])=[\pi(u), \pi(v)]$;

C3) $[u, f v]=\pi(u)(f) v+f[u, v]$;

C4) $\pi(u)\langle v, w\rangle=\langle[u, v], w\rangle+\langle v,[u, w]\rangle$;

C5) $2[u, u]=\pi^{*} d\langle u, u\rangle$.

A Courant algebroid is called regular if the anchor $\pi$ has constant rank.

Here $\pi^{*}: T^{*} M \rightarrow E$ denotes the map obtained by dualizing $\pi: E \rightarrow T M$ and identifying $E^{*}$ with $E$ using the scalar product. Therefore C5) can be written in the equivalent way

$$
2\langle[u, u], v\rangle=\pi(v)\langle u, u\rangle .
$$

Remark 2. As already pointed out in [24, 2], the axioms of a Courant algebroid can be reduced. One can show that axioms C2) and C3) from the definition of Courant algebroids follow from the other axioms. In fact, C2) can be checked by calculating $[\pi(u), \pi(v)]\langle w, e\rangle$, for any $w, e \in \Gamma(E)$, with the help of C4) and C1). Similarly, C3) can be checked by taking the scalar product with a section $w \in \Gamma(E)$ and evaluating the result with help of C4).

Example 3. The fundamental example of a Courant algebroid is the generalized tangent bundle $\mathbb{T} M=T M \oplus T^{*} M$ of a smooth manifold $M$ with the canonical projection $\mathbb{T} M \rightarrow T M$ as anchor, the scalar product defined by $\langle X+\xi, Y+\eta\rangle=\frac{1}{2}(\xi(Y)+\eta(X))$, and Dorfman bracket by

$$
[X+\xi, Y+\eta]=[X, Y]+\mathcal{L}_{X} \eta-i_{Y} d \xi+H(X, Y, \cdot)
$$

where $H \in \Omega^{3}(M)$ is a closed 3 -form and $X, Y \in \Gamma(T M), \xi, \eta \in \Gamma\left(T^{*} M\right)$. On the right-hand side, $[X, Y]$ stands for the usual Lie bracket of vector fields and $\mathcal{L}_{X} \eta$ for the Lie derivative of $\eta$ in the direction of $X$. It is known that every Courant algebroid for which the sequence $0 \rightarrow T^{*} M \stackrel{\pi^{*}}{\rightarrow} E \stackrel{\pi}{\rightarrow} T M \rightarrow 0$ is exact is isomorphic to a Courant algebroid of this form. Such Courant algebroids are called exact. 


\section{$2.2 \quad E$-connections and generalized connections}

Unless otherwise stated, $E$ will denote a Courant algebroid, with anchor $\pi: E \rightarrow T M$, scalar product $\langle\cdot, \cdot\rangle$ and Dorfman bracket $[\cdot, \cdot]$. Let $V \rightarrow M$ be a vector bundle.

Definition 4. i) An E-connection on $V$ is an $\mathbb{R}$-linear map

$$
D: \Gamma(V) \rightarrow \Gamma\left(E^{*} \otimes V\right), \quad v \mapsto D v,
$$

which satisfies the following Leibniz rule

$$
D_{e}(f v)=\pi(e)(f) v+f D_{e} v
$$

for all $e \in \Gamma(E), v \in \Gamma(V), f \in C^{\infty}(M)$, where $D_{e} v=(D v)(e)$.

ii) An E-connection on $E$ is called a generalized connection if it is compatible with the scalar product:

$$
\pi(u)\langle v, w\rangle=\left\langle D_{u} v, w\right\rangle+\left\langle v, D_{u} w\right\rangle
$$

for all $u, v, w \in \Gamma(E)$.

Example 5. A connection $\nabla$ on a vector bundle $V \rightarrow M$ induces an $E$ connection $D$ on $V$ by the formula

$$
D_{e} v:=\nabla_{\pi(e)} v
$$

for all $e \in \Gamma(E), v \in \Gamma(V)$. If $\nabla$ is a connection on $E$, then the induced $E$ connection is a generalized connection if and only if $\langle\cdot, \cdot\rangle$ is $\nabla$-parallel along $\pi(E)$, that is $\nabla_{X}\langle\cdot, \cdot\rangle=0$ for all $X \in \pi(E)$. Conversely, if the Courant algebroid $E$ is regular, an E-connection $D$ on $V$ which satisfies $D_{e}=0$ for all $e \in \operatorname{ker} \pi$ is induced by a connection $\nabla$ on $V$ and a similar statement holds for generalized connections. In fact, an E-connection $D$ on $V$ such that $D_{e}=0$ for all $e \in \operatorname{ker} \pi$ induces a partial connection $\nabla^{F}: \Gamma(F) \times \Gamma(V) \rightarrow$ $\Gamma(V)$ along the distribution $F=\pi(E) \subset T M$ such that $D_{e}=\nabla_{\pi(e)}^{F}$ for all $e \in E$. Choosing a partial connection $\nabla^{F^{\prime}}: \Gamma\left(F^{\prime}\right) \times \Gamma(V) \rightarrow \Gamma(V)$ along a complementary distribution $F^{\prime} \subset T M$, we can define a connection $\nabla$ such that $D_{e}=\nabla_{\pi(e)}$ for all $e \in E$ by $\nabla_{X+Y}=\nabla_{X}^{F}+\nabla_{Y}^{F^{\prime}}$ for all $X \in F, Y \in F^{\prime}$. If $V=E$ is a regular Courant algebroid and $D$ happens to be a generalized connection on $E$ then the partial connection $\nabla^{F}$ is metric and we can choose $\nabla^{F^{\prime}}$ (and hence $\nabla$ ) to be metric as well.

\footnotetext{
${ }^{1}$ Recall that the notion of a partial connection is defined by the same properties as that of a connection, namely tensoriality in the first argument and Leibniz rule in the second.
} 
Definition 6. The torsion $T^{D} \in \Gamma\left(\wedge^{2} E^{*} \otimes E\right)$ of a generalized connection $D$ is defined by

$$
T^{D}(u, v)=D_{u} v-D_{v} u-[u, v]+(D u)^{*} v, \forall u, v \in \Gamma(E),
$$

where $(D u)^{*}$ is the metric adjoint of $D u \in \Gamma($ End $E)$ with respect to $\langle\cdot, \cdot\rangle$.

Note that the tensoriality of $T^{D}(u, v)$ in $v$ is obvious since the operators $D_{u}$ and $[u, \cdot]$ satisfy the same Leibniz rule. The tensoriality in $u$ is a consequence of the skew-symmetry of $T^{D}$, which follows from

$$
\langle[u, v]+[v, u], w\rangle=\pi(w)\langle u, v\rangle
$$

(polarization of (1)) and the compatibility of $D$ with the scalar product. Moreover, one can check that $T^{D}(u, v, w):=\left\langle T^{D}(u, v), w\right\rangle$ is totally skewsymmetric. From (4),

$$
\langle[u, v], w\rangle=-T^{D}(u, v, w)+\left\langle D_{u} v-D_{v} u, w\right\rangle+\left\langle D_{w} u, v\right\rangle .
$$

We often identify $E$ with $E^{*}$ using the scalar product $\langle\cdot, \cdot\rangle$. By means of this identification, $\Lambda^{2} E \subset$ End $E$ is the subbundle of skew-symmetric endomorphisms of $E$, where

$$
\left(e_{1} \wedge e_{2}\right)\left(e_{3}\right):=\left\langle e_{1}, e_{3}\right\rangle e_{2}-\left\langle e_{2}, e_{3}\right\rangle e_{1}, e_{i} \in E .
$$

With this convention, any two generalized connections are related by $\tilde{D}=$ $D+\eta$, for $\eta \in \Gamma\left(E^{*} \otimes \Lambda^{2} E\right)$ and

$$
T^{\tilde{D}}(u, v, w)=T^{D}(u, v, w)+\eta(u, v, w)+\eta(w, u, v)+\eta(v, w, u) .
$$

The space of torsion-free generalized connections is non-empty, see [10]. If $D$ and $\tilde{D}=D+\eta$ are torsion-free (or, more generally, have the same torsion), then $\eta$ is of the form

$$
\eta(u, v, w)=\sigma(u, v, w)-\sigma(u, w, v),
$$

for a section $\sigma \in \Gamma\left(S^{2} E \otimes E\right)$, see [10].

\subsection{Generalized metrics}

Let $E$ be a Courant algebroid with anchor $\pi: E \rightarrow T M$, scalar product $\langle\cdot, \cdot\rangle$ and Dorfmann bracket $[\cdot, \cdot]$. 
Definition 7. A generalized metric on $E$ is a vector subbundle $E_{+}$of $E$ on which $\langle\cdot, \cdot\rangle$ is non-degenerate.

Let $E_{-}:=E_{+}^{\perp}$ be the orthogonal complement of $E_{+}$with respect to $\langle\cdot, \cdot\rangle$. Then $G:=\left.\langle\cdot, \cdot\rangle\right|_{E_{+} \times E_{+}}-\left.\langle\cdot, \cdot\rangle\right|_{E_{-} \times E_{-}}$is a non-degenerate metric on $E$. Alternatively, a generalized metric on $E$ can be defined as a non-degenerate symmetric bilinear form $G$ on the vector bundle $E$ such that the endomorphism $G^{\text {end }}$, defined by $G(u, v)=\left\langle G^{\text {end }} u, v\right\rangle$, satisfies $\left(G^{\text {end }}\right)^{2}=\operatorname{Id}_{E}$. The bundles $E_{ \pm}$are the \pm 1 -eigenbundles of $G^{\text {end }}$. Along the paper we denote by $e_{ \pm}:=\frac{1}{2}\left(\operatorname{Id} \pm G^{\text {end }}\right) e$ the $E_{ \pm}$-components of a vector $e \in E$ in the decomposition $E=E_{+} \oplus E_{-}$determined by a generalized metric $G$.

Given a generalized metric $G$ there is always a torsion-free generalized connection which preserves $G$ (see [13, 10]). Such a connection is not unique if rank $E>1$. It is called a Levi-Civita connection of $G$. The non-uniqueness is due to the fact that although the first prolongation $\mathfrak{s o}(k, \ell)^{(1)}$ is always trivial (which is responsible for the uniqueness of the Levi-Civita connection of a pseudo-Riemannian manifold), the generalized first prolongation $\mathfrak{s o}(k, \ell)^{\langle 1\rangle}$ (see Definition 16 below) is non-trivial if $k+\ell>1$.

\subsection{Generalized complex and hyper-complex structures}

Definition 8. A generalized almost complex structure on $E$ is an endomorphism $\mathcal{J} \in \Gamma($ End $E)$ which satisfies $\mathcal{J}^{2}=-\operatorname{Id}_{E}$ and is orthogonal with respect to the scalar product $\langle\cdot, \cdot\rangle$ of $E$. We say that $\mathcal{J}$ is integrable (or is a generalized complex structure) if its Nijenhuis tensor

$$
N_{\mathcal{J}}(u, v):=[\mathcal{J} u, \mathcal{J} v]-[u, v]-\mathcal{J}([\mathcal{J} u, v]+[u, \mathcal{J} v])
$$

vanishes identically.

The following simple lemma will be useful for us.

Lemma 9. Let $\mathcal{J}$ be a generalized almost complex structure on $E$. Then $N_{\mathcal{J}}(u, v, w):=\left\langle N_{\mathcal{J}}(u, v), w\right\rangle$ is a 3-form on $E$.

Proof. The skew-symmetry of $N_{\mathcal{J}}(u, v, w)$ in the first two arguments follows from (5). In order to prove the skew-symmetry of $N_{\mathcal{J}}(u, v, w)$ in the last two arguments we compute

$$
\begin{aligned}
& N_{\mathcal{J}}(u, v, w)+N_{\mathcal{J}}(u, w, v) \\
& =\langle[\mathcal{J} u, \mathcal{J} v]-[u, v], w\rangle+\langle[\mathcal{J} u, v]+[u, \mathcal{J} v], \mathcal{J} w\rangle \\
& +\langle[\mathcal{J} u, \mathcal{J} w]-[u, w], v\rangle+\langle[\mathcal{J} u, w]+[u, \mathcal{J} w], \mathcal{J} v\rangle \\
& =\langle[\mathcal{J} u, \mathcal{J} v], w\rangle+\langle\mathcal{J} v,[\mathcal{J} u, w]\rangle-(\langle[u, v], w\rangle+\langle[u, w], v\rangle) \\
& +\langle[\mathcal{J} u, v], \mathcal{J} w\rangle+\langle[\mathcal{J} u, \mathcal{J} w], v\rangle+\langle[u, \mathcal{J} v], \mathcal{J} w\rangle+\langle[u, \mathcal{J} w], \mathcal{J} v\rangle .
\end{aligned}
$$


Using the axiom C4) from the definition of Courant algebroids we obtain

$$
\begin{aligned}
& N_{\mathcal{J}}(u, v, w)+N_{\mathcal{J}}(u, w, v) \\
& =\pi(\mathcal{J} u)\langle\mathcal{J} v, w\rangle-\pi(u)\langle v, w\rangle+\pi(\mathcal{J} u)\langle v, \mathcal{J} w\rangle+\pi(u)\langle\mathcal{J} v, \mathcal{J} w\rangle=0 .
\end{aligned}
$$

We have proved that $N_{\mathcal{J}}=N_{\mathcal{J}}(u, v, w)$ is completely skew-symmetric. As it is $C^{\infty}(M)$-linear in $w$, it is $C^{\infty}(M)$-linear also in $u, v$.

Definition 10. A generalized almost hypercomplex structure on $E$ is a triple $\left(\mathcal{J}_{1}, \mathcal{J}_{2}, \mathcal{J}_{3}\right)$ of anti-commuting generalized almost complex structures such that $\mathcal{J}_{3}=\mathcal{J}_{1} \mathcal{J}_{2}$. We say that $\left(\mathcal{J}_{1}, \mathcal{J}_{2}, \mathcal{J}_{3}\right)$ is integrable (is a generalized hypercomplex structure) if all $\mathcal{J}_{i}$ are generalized (integrable) complex structures.

\subsection{Generalized Kähler and hyper-Kähler structures}

Definition 11. A generalized almost Hermitian structure on $E$ is a pair $(G, \mathcal{J})$ where $G$ is a generalized metric and $\mathcal{J}$ a generalized almost complex structure on $E$, such that $G(\mathcal{J} u, \mathcal{J} v)=G(u, v)$ for all $u, v \in E$.

The endomorphism $G^{\text {end }}$ of $E$ determined by $G$ (see Section 2.3) commutes with $\mathcal{J}_{1}:=\mathcal{J}$ and $\mathcal{J}_{2}:=G^{\text {end }} \mathcal{J}$ is a generalized almost complex structure. The generalized almost complex structures $\mathcal{J}_{1}$ and $\mathcal{J}_{2}$ preserve $E_{ \pm}\left(\right.$the \pm 1 -eigenbundles of $\left.G^{\text {end }}\right)$. Moreover, $\mathcal{J}_{2}= \pm \mathcal{J}_{1}$ on $E_{ \pm}$.

Definition 12. A generalized almost Hermitian structure $(G, \mathcal{J})$ is called a generalized Kähler structure if $\mathcal{J}_{1}=\mathcal{J}$ and $\mathcal{J}_{2}=G^{\text {end }} \mathcal{J}_{1}$ are integrable.

The argument from Proposition 2.17 of [17] works also in the setting of non-exact Courant algebroids and shows that $(G, \mathcal{J})$ is a generalized Kähler structure if and only if $L \cap\left(E_{ \pm}\right)_{\mathbb{C}}$ are closed under the Dorfmann bracket $[\cdot, \cdot]$ of $E$, where $L$ denotes the $(1,0)$-bundle of $\mathcal{J}$.

Definition 13. i) A generalized almost hyper-Hermitian structure is a generalized almost hypercomplex structure $\left(\mathcal{J}_{1}, \mathcal{J}_{2}, \mathcal{J}_{3}\right)$ together with a generalized metric $G$, such that $\left(G, \mathcal{J}_{i}\right)$ is a generalized almost Hermitian structure, for all $i=1,2,3$.

ii) A generalized almost hyper-Hermitian structure $\left(G, \mathcal{J}_{1}, \mathcal{J}_{2}, \mathcal{J}_{3}\right)$ is a generalized hyper-Kähler structure if $\left(G, \mathcal{J}_{i}\right)$ is a generalized Kähler structure, for all $i=1,2,3$. 


\subsection{Intrinsic torsion of a generalized structure}

Given a linear Lie group $H \subset \mathrm{O}(k, \ell)$, a generalized $H$-structure on a Courant algebroid $E$ with scalar product $\langle\cdot, \cdot\rangle$ of signature $(k, \ell)$ is a reduction of the structure group of $E$ from $\mathrm{O}(k, \ell)$ to $H$.

Let $\left(T_{i}^{0}\right)$ be a system of tensors on $\mathbb{R}^{k+\ell}$ and $Q=\left(T_{i}\right)$ a system of tensor fields on $E$, such that $T_{i}$ is pointwise linearly equivalent to $T_{i}^{0}$. Then we associate to $Q$ the generalized $H$-structure defined as the principal bundle of standard orthonormal frames of $E$ with respect to which each of the tensor fields $T_{i}$ takes the constant form $T_{i}^{0}$ and the scalar product is represented by the matrix $\operatorname{diag}\left(\mathbf{1}_{k},-\mathbf{1}_{\ell}\right)$. Examples considered in this paper are:

1. generalized almost complex structures $Q=\mathcal{J}, H=\mathrm{U}\left(\frac{k}{2}, \frac{\ell}{2}\right)$,

2. generalized almost hypercomplex structures $Q=\left(\mathcal{J}_{1}, \mathcal{J}_{2}, \mathcal{J}_{3}\right), H=$ $\operatorname{Sp}\left(\frac{k}{4}, \frac{\ell}{4}\right)$,

3. generalized Riemannian metrics $Q=G, H=\mathrm{O}\left(k_{+}, l_{+}\right) \times \mathrm{O}\left(k_{-}, l_{-}\right)$, where $\left.\langle\cdot, \cdot\rangle\right|_{E_{ \pm}}$has signature $\left(k_{ \pm}, \ell_{ \pm}\right)$(in particular, $k_{+}+k_{-}=k$ and $\left.\ell_{+}+\ell_{-}=\ell\right)$,

4. generalized almost Hermitian structures $Q=(G, \mathcal{J}), H=\mathrm{U}\left(\frac{k_{+}}{2}, \frac{\ell_{+}}{2}\right) \times$ $\mathrm{U}\left(\frac{k_{-}}{2}, \frac{\ell_{-}}{2}\right)$, where $\left.\langle\cdot, \cdot\rangle\right|_{E_{ \pm}}$has signature $\left(k_{ \pm}, \ell_{ \pm}\right)$, and

5. generalized almost hyper-Hermitian structures $Q=\left(G, \mathcal{J}_{1}, \mathcal{J}_{2}, \mathcal{J}_{3}\right)$, $H=\operatorname{Sp}\left(\frac{k_{+}}{4}, \frac{\ell_{+}}{4}\right) \times \operatorname{Sp}\left(\frac{k_{-}}{4}, \frac{\ell_{-}}{4}\right)$.

We will refer to these simply as generalized $H$-structures $Q$.

In analogy with the classical case (see e.g. [23]), given a generalized $\mathrm{H}$ structure $Q$ on $E$ we may consider the space of generalized connections $D$ which preserve, or are adapted, to $Q$ (i.e. $D Q=0$ ). A generalized connection adapted to a generalized almost complex (respectively, hypercomplex) structure $Q=\mathcal{J}$ (respectively, $Q=\left(\mathcal{J}_{1}, \mathcal{J}_{2}, \mathcal{J}_{3}\right)$ ) will be called complex (respectively, hypercomplex).

Lemma 14. Any generalized $H$-structure $Q$ on a Courant algebroid E admits an adapted generalized connection.

Proof. Any connection on the principal $H$-bundle of frames which defines the generalized $H$-structure induces a connection on $E$ for which $Q$ and the scalar product $\langle\cdot, \cdot\rangle$ are parallel. This connection extends as in Example 5 to a generalized connection adapted to $Q$. 
The space of generalized connections adapted to a generalized $H$-structure $Q$ is an affine space, modelled on the vector space of sections of the bundle $E^{*} \otimes \operatorname{ad}_{Q}(E)$, where $\operatorname{ad}_{Q}(E)$ denotes the bundle of 2-forms $\alpha \in \Lambda^{2} E^{*}$, adapted to $Q$. By the latter condition we mean that $\operatorname{ad}_{\alpha} \in \operatorname{End}\left(\mathcal{T}_{E}\right)$, defined as the natural extension of the action of $\alpha \in \Lambda^{2} E^{*} \cong \mathfrak{s o}(E) \subset \operatorname{End}(E)$ to the tensor bundle $\mathcal{T}_{E}=\oplus_{p, q} E^{p} \otimes\left(E^{*}\right)^{q}$ of $E$, annihilates the tensor fields from $Q$. When $Q$ belongs to the above list, we require that $[\alpha, \mathcal{J}]=0$ and $\left[\alpha, G^{\text {end }}\right]=0$ when $\mathcal{J}, G \in Q$. These conditions are equivalent to $\mathcal{J}^{*} \alpha=\alpha$ and $\left(G^{\text {end }}\right)^{*} \alpha=\alpha$. Note that the fiber $\operatorname{ad}_{Q}(E)_{x}, x \in M$, of the $\operatorname{bundle}_{Q} \operatorname{ad}_{Q}(E) \rightarrow M$ is a Lie algebra isomorphic to the Lie algebra $\mathfrak{h} \subset \mathfrak{s o}(k, \ell)$ of the structure group $H$. The map

$\partial_{Q}: E^{*} \otimes \operatorname{ad}_{Q}(E) \rightarrow \Lambda^{3} E^{*},\left(\partial_{Q} \eta\right)(u, v, w):=\eta(u, v, w)+\eta(w, u, v)+\eta(v, w, u)$

is called the algebraic torsion map. From (7), the image of $T^{D} \in \Gamma\left(\Lambda^{3} E^{*}\right)$ in the quotient bundle $\left(\Lambda^{3} E^{*}\right) /$ im $\partial_{Q}$ is independent of the choice of adapted generalized connection $D$.

Definition 15. Let $Q$ be a generalized $H$-structure on $E$ and $D$ an adapted generalized connection. The class of $T^{D}$ in $\left(\Lambda^{3} E^{*}\right) / \mathrm{im} \partial_{Q}$ is called the intrinsic torsion of $Q$.

We shall often consider the intrinsic torsion as a 3 -form on $E$, by choosing a suitable complement $C(E)$ of im $\partial_{Q}$ in $\Lambda^{3} E^{*}$ and identifying the quotient $\left(\Lambda^{3} E^{*}\right) / \operatorname{im} \partial_{Q}$ with $C(E)$.

Definition 16. The generalized first prolongation of a Lie algebra $\mathfrak{h} \subset \mathfrak{s o}(k, \ell)$ $\cong \Lambda^{2} V^{*}, V:=\mathbb{R}^{k+\ell}$, is the subspace

$$
\mathfrak{h}^{\langle 1\rangle}:=\left\{\eta \in V^{*} \otimes \mathfrak{h} \mid \partial \eta=0\right\} \subset V^{*} \otimes \Lambda^{2} V^{*},
$$

where $\partial: V^{*} \otimes \Lambda^{2} V^{*} \rightarrow \Lambda^{3} V^{*}$ is defined by:

$$
(\partial \eta)(u, v, w):=\eta(u, v, w)+\eta(w, u, v)+\eta(v, w, u), \quad u, v, w \in V .
$$

Proposition 17. Let $Q$ be a generalized H-structure on a Courant algebroid $E$ and $D_{0}$ an adapted generalized connection with torsion $T^{D_{0}}$. Given a section $T \in \Gamma\left(\Lambda^{3} E^{*}\right)$ there exists a generalized connection $D$ adapted to $Q$ with torsion $T^{D}=T$ if and only if $T-T^{D_{0}} \in \Gamma\left(\mathrm{im} \partial_{Q}\right)$. The generalized connection $D$ is unique up to addition of a section of $\operatorname{ker} \partial_{Q} \subset E^{*} \otimes \operatorname{ad}_{Q}(E)$. It is unique if and only if the generalized first prolongation $\mathfrak{h}^{\langle 1\rangle}$ of the Lie algebra $\mathfrak{h} \subset \mathfrak{s o}(k, \ell)$ of the structure group $H$ vanishes. 
Proof. This is obtained by writing an arbitrary adapted generalized connection as $D=D_{0}+\eta$, where $\eta \in \Gamma\left(E^{*} \otimes \operatorname{ad}_{Q}(E)\right)$ and observing that $T^{D}=T^{D_{0}}+\partial_{Q} \eta$, see (7). The last statement follows from the fact that the fibres of the bundle ker $\partial_{Q}$ are isomorphic to $\mathfrak{h}^{\langle 1\rangle}$.

The next corollary follows easily from Proposition 17.

Corollary 18. In the setting of Proposition 17, $Q$ admits an adapted torsionfree generalized connection $D$ if and only if the intrinsic torsion of $Q$ vanishes. The generalized connection $D$ is unique if and only if $\mathfrak{h}^{\langle 1\rangle}=0$.

\subsection{Application to Born geometry}

The vanishing of the generalized first prolongation for certain Lie subalgebras of $\mathfrak{s o}(k, \ell)$ can be used to prove the uniqueness of certain connections (rather than generalized connections). As an example we mention the canonical connection in Born geometry defined in [9], which is related to string compactifications and more specifically to double field theory. Its uniqueness, proven in [9], can be alternatively deduced from the vanishing of the generalized first prolongation of the diagonal $\mathfrak{s o}(n)$-subalgebra

$$
\Delta_{\mathfrak{s o}(n)}=\{(A, A) \in \mathfrak{s o}(n) \oplus \mathfrak{s o}(n) \mid A \in \mathfrak{s o}(n)\} \subset \mathfrak{s o}(n, n) .
$$

The corresponding diagonally embedded $\mathrm{O}(n)$-subgroup $\Delta_{\mathrm{O}(n)} \subset \mathrm{O}(n, n)$ is precisely the automorphism group of the following data on $V=\mathbb{R}^{2 n}$ :

1. a scalar product $\eta=\langle\cdot, \cdot\rangle$ of neutral signature,

2. a positive definite scalar product $g$ and

3. a linear involution $K$,

which satisfy the following compatibility conditions:

1. $K$ is skew-symmetric with respect to $\eta$,

2. $J=\eta^{-1} g$ is an involution and

3. $J$ anti-commutes with $K$.

These properties imply that the triple $(I:=K J, J, K)$ is a para-hypercomplex structure on $V$, i.e. $I, J, K$ pairwise anti-commute, $K=I J$ and $-I^{2}=J^{2}=$ $K^{2}=\mathrm{Id}$. However, the structure is not para-hyper-Hermitian with respect to $\eta$ as only $K$ is skew-symmetric, whereas $I$ and $J$ are symmetric with respect to $\eta$. A quadruple $(\eta, I, J, K)$ with these properties is called a Born structure 
on $V$ if the symmetric bilinear form $g=\eta J=\eta(J \cdot, \cdot)$ is positive definite. So the data $(\eta, g, K)$ with the above compatibility relations 1.-3. is equivalent to a Born structure $(\eta, I, J, K)$ on $V$.

Given smooth tensor fields $(\eta, I, J, K)$ on a manifold $M$ such that $\left(\eta_{p}, I_{p}, J_{p}\right.$, $\left.K_{p}\right)$ is a Born structure on $T_{p} M$ for all $p \in M$, the data $(\eta, I, J, K)$ is called a Born structure on $M$. It is proven in [9] that every Born structure $(\eta, I, J, K)$ on a manifold $M$ admits a canonical compatible connection $\nabla$, called the Born connection, with vanishing generalized torsion $\mathcal{T}_{\nabla} \in \Omega^{3}(M)$, where

$$
\mathcal{T}_{\nabla}(X, Y, Z):=\eta\left(\nabla_{X} Y-\nabla_{Y} X-[X, Y]^{c}+(\nabla X)^{*} Y, Z\right)
$$

is defined in terms of the so-called canonical D-bracket

$$
[X, Y]^{c}=\nabla_{X}^{c} Y-\nabla_{Y}^{c} X+\left(\nabla^{c} X\right)^{*} Y .
$$

Here $B^{*}$ denotes the $\eta$-adjoint of an endomorphism field $B$ and $\nabla^{c}:=$ $\nabla^{\eta}+\frac{1}{2} K \nabla^{\eta} K$ is the canonical connection compatible with the almost paraHermitian structure $(\eta, K)$, where $\nabla^{\eta}$ denotes the Levi-Civita connection of $\eta$. The Born connection is denoted $\nabla^{B}$ and is defined by

$$
\nabla_{X}^{B} Y:=\left[X_{-}, Y_{+}\right]_{+}^{c}+\left[X_{+}, Y_{-}\right]_{-}^{c}+\left(K\left[X_{+}, K Y_{+}\right]^{c}\right)_{+}+\left(K\left[X_{-}, K Y_{-}\right]^{c}\right)_{-},
$$

where \pm stands for the projections onto the eigendistributions of $J$. It is clear that any two connections $\nabla^{\prime}$ and $\nabla$ compatible with the same Born structure and having the same generalized torsion differ by a section $A \in$ $\Gamma\left(T^{*} M \otimes \mathfrak{s o}(T M)\right)$ in the kernel of the total skew-symmetrization map $\partial$, such that $A_{p}$ belongs to the generalized first prolongation of the Lie algebra $\operatorname{aut}\left(T_{p} M, \eta_{p}, I_{p} . J_{p}, K_{p}\right) \cong \Delta_{\mathfrak{s o}(n)}$. This shows, in particular, that the uniqueness of $\nabla^{B}$ follows from the next lemma.

Lemma 19. $\Delta_{\mathfrak{s o}(n)}^{\langle 1\rangle}=0$.

Proof. Let $\left(e_{1}, \ldots, e_{n}, e_{1}^{\prime}, \ldots, e_{n}^{\prime}\right)$ be a basis of $V$, orthonormal with respect to both $\langle\cdot, \cdot\rangle$ and $g$, where

$$
\left\langle e_{i}, e_{i}\right\rangle=g\left(e_{i}, e_{i}\right)=1,\left\langle e_{i}^{\prime}, e_{i}^{\prime}\right\rangle=-g\left(e_{i}^{\prime}, e_{i}^{\prime}\right)=-1,
$$

such that $K\left(e_{i}\right)=e_{i}^{\prime}, K\left(e_{i}^{\prime}\right)=e_{i}$. Then $J\left(e_{i}\right)=e_{i}$ and $J\left(e_{i}^{\prime}\right)=-e_{i}^{\prime}$, from where we deduce that $A_{v}$ preserves $\operatorname{span}\left\{e_{1}, \ldots, e_{n}\right\}$ and $\operatorname{span}\left\{e_{1}^{\prime}, \ldots, e_{n}^{\prime}\right\}$, for any $A \in V^{*} \otimes \Delta_{\mathfrak{s o}(n)}$ and $v \in V$ (since $A_{v}$ commutes with $J$ ). We deduce that $A$ is completely determined by two $(1,2)$-tensors $\left(A_{i j}^{k}\right)$ and $\left(A_{i j}^{\prime k}\right)$ on $\mathbb{R}^{n}$, where $A_{e_{i}} e_{j}=\sum_{k} A_{i j}^{k} e_{k}$ and $A_{e_{i}^{\prime}} e_{j}=\sum_{k} A_{i j}^{\prime k} e_{k}$, since (from $A_{v} K=K A_{v}$ for any $v) A_{e_{i}} e_{j}^{\prime}=\sum_{k} A_{i j}^{k} e_{k}^{\prime}$ and $A_{e_{i}^{\prime}} e_{j}^{\prime}=\sum_{k} A_{i j}^{\prime k} e_{k}^{\prime}$. From $\partial A=0$ we obtain

$$
0=\left\langle A_{e_{i}} e_{j}^{\prime}, e_{k}^{\prime}\right\rangle+\left\langle A_{e_{j}^{\prime}} e_{k}^{\prime}, e_{i}\right\rangle+\left\langle A_{e_{k}^{\prime}} e_{i}, e_{j}^{\prime}\right\rangle=-A_{i j}^{k}
$$

and similarly $A_{i j}^{\prime k}=0$. This proves that $A=0$. 


\section{Generalized almost complex structures}

\subsection{Intrinsic torsion of a generalized almost complex structure}

In this section we compute the intrinsic torsion $t_{\mathcal{J}}$ of a generalized almost complex structure $\mathcal{J}$ on a Courant algebroid $E$ and relate it to the Nijenhuis tensor $N_{\mathcal{J}}$. Before we need to recall basic facts on projectors. Recall that an endomorphism $P \in \Gamma$ (End $V$ ) of a vector bundle $V$ is a projector onto a subbundle $V_{0} \subset V$ if $P^{2}=P$ and $\operatorname{im} P=V_{0}$. Then $V$ decomposes as $V=$ $V_{0} \oplus$ ker $P$ and $P$ (respectively $\mathrm{Id}-P$ ) are the projections onto $V_{0}$ (respectively ker $P$ ) along this decomposition. In particular, there is a canonical choice of a complement of im $P$ in $V$, namely, ker $P$.

Consider now the algebraic torsion map $\partial_{\mathcal{J}}: E^{*} \otimes \Lambda_{\mathcal{J}}^{1,1} E^{*} \rightarrow \Lambda^{3} E^{*}$ of $\mathcal{J}$, defined by (9), where $\operatorname{ad}_{\mathcal{J}}(E)=\Lambda_{\mathcal{J}}^{1,1} E^{*}$ is the bundle of $\mathcal{J}$-invariant 2-forms on $E$.

Lemma 20. i) The map $\Pi_{\mathcal{J}} \in \Gamma\left(\operatorname{End}\left(\Lambda^{3} E^{*}\right)\right)$ defined by

$$
\begin{gathered}
\left(\Pi_{\mathcal{J}} \alpha\right)(u, v, w):= \\
\frac{1}{4}(\alpha(u, v, w)-\alpha(u, \mathcal{J} v, \mathcal{J} w)-\alpha(\mathcal{J} u, v, \mathcal{J} w)-\alpha(\mathcal{J} u, \mathcal{J} v, w))
\end{gathered}
$$

is a projector onto the subbundle

$$
\Lambda_{\mathcal{J}}^{3} E^{*}:=\left\{\alpha \in \Lambda^{3} E^{*}, \alpha(\mathcal{J} u, v, w)=\alpha(u, \mathcal{J} v, w)=\alpha(u, v, \mathcal{J} w)\right\} .
$$

ii) The equality $\operatorname{im} \partial_{\mathcal{J}}=\operatorname{ker} \Pi_{\mathcal{J}}$ holds.

Proof. It is straightforward to check that $\operatorname{im} \Pi_{\mathcal{J}} \subset \Lambda_{\mathcal{J}}^{3} E^{*}$. Also, for all $\alpha \in \Lambda_{\mathcal{J}}^{3} E^{*}, \Pi_{\mathcal{J}}(\alpha)=\alpha$. We obtain that $\operatorname{im} \Pi_{\mathcal{J}}=\Lambda_{\mathcal{J}}^{3} E^{*}$ and $\Pi_{\mathcal{J}}^{2}=\Pi_{\mathcal{J}}$. Claim i) is proved. To prove claim ii), we notice that $\Pi_{\mathcal{J}} \circ \partial_{\mathcal{J}}=0$, i.e. $\operatorname{im} \partial_{\mathcal{J}} \subset \operatorname{ker} \Pi_{\mathcal{J}}$. Let

$$
\tilde{\pi}_{\mathcal{J}}: \Lambda^{3} E^{*} \rightarrow E^{*} \otimes \Lambda_{\mathcal{J}}^{1,1} E^{*},\left(\tilde{\pi}_{\mathcal{J}} \alpha\right)(u, v, w):=\alpha(u, v, w)+\alpha(u, \mathcal{J} v, \mathcal{J} w) .
$$

It is straightforward to check that $\Pi_{\mathcal{J}}=\operatorname{Id}_{\Lambda^{3} E^{*}}-\frac{1}{4} \partial_{\mathcal{J}} \circ \tilde{\pi}_{\mathcal{J}}$, which implies $\operatorname{ker} \Pi_{\mathcal{J}} \subset \operatorname{im} \partial_{\mathcal{J}}$. We proved that $\operatorname{im} \partial_{\mathcal{J}}=\operatorname{ker} \Pi_{\mathcal{J}}$.

The next corollary follows from Lemma 20 and our comments before this lemma.

Corollary 21. With the notation from Lemma 20, $\operatorname{im} \Pi_{\mathcal{J}}$ is a complement of $\operatorname{im} \partial_{\mathcal{J}}$ in $\Lambda^{3} E^{*}$. 
From Corollary 21, we can (and will) identify the quotient $\left(\Lambda^{3} E^{*}\right) /\left(\operatorname{im} \partial_{\mathcal{J}}\right)$ with $\operatorname{im} \Pi_{\mathcal{J}}=\Lambda_{\mathcal{J}}^{3} E^{*}$ and consider the intrinsic torsion $t_{\mathcal{J}}$ of $\mathcal{J}$ as a section of $\Lambda_{\mathcal{J}}^{3} E^{*}$. On the other hand, $N_{\mathcal{J}}(\mathcal{J} u, v)=-\mathcal{J} N_{\mathcal{J}}(u, v)$ (easy check), which implies that $N_{\mathcal{J}}$, considered as a 3 -form (see Lemma 9), is also a section of $\Lambda_{\mathcal{J}}^{3} E^{*}$. Up to a constant, $N_{\mathcal{J}}$ and $t_{\mathcal{J}}$ coincide. More precisely, we have the following result.

Corollary 22. The torsion $T^{D}$ of a generalized connection $D$ with $D \mathcal{J}=0$ satisfies

$T^{D}(u, v, w)-T^{D}(\mathcal{J} u, v, \mathcal{J} w)-T^{D}(u, \mathcal{J} v, \mathcal{J} w)-T^{D}(\mathcal{J} u, \mathcal{J} v, w)=N_{\mathcal{J}}(u, v, w)$,

for all $u, v, w \in \Gamma(E)$. In particular, $t_{\mathcal{J}}=\frac{1}{4} N_{\mathcal{J}}$ (viewed as 3 -forms on $E$ ).

Proof. Relation (11) follows from (8), together with

$$
[u, v]=D_{u} v-D_{v} u+(D u)^{*} v-T^{D}(u, v)
$$

and $D \mathcal{J}=0$. Relation (11) can be written as

$$
\Pi_{\mathcal{J}}\left(T^{D}\right)=\frac{1}{4} N_{\mathcal{J}}
$$

which implies the second statement.

\subsection{Integrability using torsion-free generalized connec- tions}

In this section we prove the following theorem.

Theorem 23. A generalized almost complex structure $\mathcal{J}$ on $E$ is integrable if and only if there is a torsion-free generalized connection $D$ such that $D \mathcal{J}=0$.

Part of the statement of Theorem 23 follows from Corollary 22, if there is a torsion-free generalized connection $D$ such that $D \mathcal{J}=0$ then from relation (11) $\mathcal{J}$ is integrable. For the converse statement, let $\mathcal{J} \in \Gamma(\operatorname{End} E)$ be a generalized almost complex structure. We will construct a generalized complex connection whose torsion equals the intrinsic torsion of $\mathcal{J}$. Such a generalized connection will be torsion-free (and complex) when $\mathcal{J}$ is integrable. This will conclude the proof of Theorem 23. The next remark represents our motivation for the choice of generalized connection $\tilde{D}$ in Proposition 25. 
Remark 24. Given an almost complex structure $J$ and a torsion-free connection $\nabla$ on a manifold $M$, the connection

$$
\tilde{\nabla}_{X} Y=\nabla_{X} Y-\frac{1}{4}\{(\nabla J) X, J\} Y-\frac{1}{2} J\left(\nabla_{X} J\right) Y,
$$

where $\{A, B\}:=A B+B A$ denotes the anti-commutator of $A$ and $B$ and $(\nabla J) X \in \Gamma(\operatorname{End} T M)$ is defined by $Y \rightarrow\left(\nabla_{Y} J\right) X$, is complex $(\tilde{\nabla} J=0)$ and its torsion satisfies $T^{\tilde{\nabla}}(X, Y)=\frac{1}{8} N_{J}(X, Y)$ (see Theorem 3.4 of [20]; remark the difference by a multiplicative factor between our definition for the Nijenhuis tensor and that of [20]). In particular, if $J$ is integrable, then $\tilde{\nabla}$ is torsion-free (and complex). Now, for a generalized almost complex structure $\mathcal{J}$ and a generalized torsion-free connection $D$ on the Courant algebroid $E$, we may define the analogous expression

$$
\tilde{D}_{u}^{\prime} v=D_{u} v-\frac{1}{4}\{(D \mathcal{J}) u, \mathcal{J}\} v-\frac{1}{2} \mathcal{J}\left(D_{u} \mathcal{J}\right) v
$$

However, $\tilde{D}^{\prime}$ defined by (15) is not a generalized connection (while $\mathcal{J} D_{u} \mathcal{J}$ is skew-symmetric with respect to the scalar product $\langle\cdot, \cdot\rangle$ of $E$, the anticommutator $\{(D \mathcal{J}) u, \mathcal{J}\}$ is not and $\tilde{D}^{\prime}$ does not preserve $\langle\cdot, \cdot\rangle$, in general; we shall give more details on this argument in Lemma 26). In the next lemma we modify $\tilde{D}^{\prime}$ in order to obtain a generalized connection. It will turn out that it has the required properties.

Proposition 25. Let $\mathcal{J}$ be a generalized almost complex structure and $D$ a torsion-free generalized connection on E. Define

$$
\tilde{D}_{u} v=D_{u} v-\frac{1}{4}\left\{A_{u}^{\mathrm{sym}}, \mathcal{J}\right\} v-\frac{1}{2} \mathcal{J}\left(D_{u} \mathcal{J}\right) v
$$

where $A_{u}:=(D \mathcal{J}) u$ and $A_{u}^{\mathrm{sym}}$ is its $\langle\cdot, \cdot\rangle$-symmetric part. Then $\tilde{D}$ is a generalized connection, which preserves $\mathcal{J}$. Its torsion is given by

$$
T^{\tilde{D}}(u, v, w)=\frac{1}{4} N_{\mathcal{J}}(u, v, w) .
$$

In particular, if $\mathcal{J}$ is integrable, then $\tilde{D}$ is torsion-free (and complex).

We divide the proof of the above proposition into several lemmas.

Lemma 26. Equation (16) defines a generalized complex connection.

Proof. Note that $\mathcal{J} D_{u} \mathcal{J}$ is skew-symmetric with respect to the scalar product $\langle\cdot, \cdot\rangle$ of $E\left(D_{u} \mathcal{J}\right.$ is skew-symmetric and also $\mathcal{J} D_{u} \mathcal{J}$ is skew-symmetric, being 
the composition of two anti-commuting skew-symmetric endomorphisms). Similarly, $\left\{A_{u}^{\text {sym }}, \mathcal{J}\right\}$ is skew-symmetric, because $A_{u}^{\text {sym }}$ is symmetric and $\mathcal{J}$ is skew-symmetric. We obtain that $\tilde{D}_{u}$ and $D_{u}$ differ by a skew-symmetric endomorphism, i.e. $\tilde{D}$ is a generalized connection. The generalized connection

$$
D_{u}^{(1)}:=D_{u}-\frac{1}{2} \mathcal{J} D_{u} \mathcal{J}
$$

preserves $\mathcal{J}$. As $\left\{A_{u}^{\text {sym }}, \mathcal{J}\right\}$ commutes with $\mathcal{J}$, we obtain that

$$
\tilde{D}_{u}=D_{u}^{(1)}-\frac{1}{4}\left\{A_{u}^{\mathrm{sym}}, \mathcal{J}\right\}
$$

preserves $\mathcal{J}$ as well.

In the next lemmas we prove relation (17). From (19),

$$
\begin{aligned}
\left\langle\tilde{D}_{u} v, w\right\rangle=\left\langle D_{u}^{(1)} v, w\right\rangle & -\frac{1}{8}(\eta(\mathcal{J} v, u, w)+\eta(w, u, \mathcal{J} v)) \\
& +\frac{1}{8}(\eta(v, u, \mathcal{J} w)+\eta(\mathcal{J} w, u, v)),
\end{aligned}
$$

where $\eta=\eta^{D, \mathcal{J}}$ is defined by

$$
\eta(u, v, w):=\left\langle\left(D_{u} \mathcal{J}\right) v, w\right\rangle .
$$

Remark that $\eta$ has the symmetries

$$
\eta(u, v, w)=-\eta(u, w, v), \eta(u, \mathcal{J} v, w)=\eta(u, v, \mathcal{J} w) .
$$

Lemma 27. The torsion of $D^{(1)}$ is given by

$$
T^{D^{(1)}}(u, v, w)=\frac{1}{2} \sum_{(u, v, w) \text { cyclic }} \eta(u, v, \mathcal{J} w)
$$

where the sum is over cyclic permutations on $(u, v, w)$.

Proof. The claim follows from the torsion-free property of $D$ together with relations (7) and (18).

Lemma 28. The following relation holds:

$$
N_{\mathcal{J}}(u, v, w)=\sum_{(u, v, w) \text { cyclic }}(\eta(u, v, \mathcal{J} w)+\eta(\mathcal{J} u, v, w))
$$


Proof. Using relations (8), (12) and $T^{D}=0$, we obtain

$$
\begin{aligned}
N_{\mathcal{J}}(u, v) & =\left(D_{\mathcal{J} u} \mathcal{J}\right) v-\left(D_{\mathcal{J} v} \mathcal{J}\right) u-\mathcal{J}\left(D_{u} \mathcal{J}\right) v \\
& +\mathcal{J}\left(D_{v} \mathcal{J}\right) u+(D(\mathcal{J} u))^{*} \mathcal{J} v-(D u)^{*} v \\
& -\mathcal{J}(D(\mathcal{J} u))^{*} v-\mathcal{J}(D u)^{*} \mathcal{J} v
\end{aligned}
$$

Taking the inner product of the above equality with $w$ and using the symmetries (22) of $\eta$ we obtain

$$
\begin{aligned}
N_{\mathcal{J}}(u, v, w)= & \left\langle N_{\mathcal{J}}(u, v), w\right\rangle=\eta(\mathcal{J} u, v, w)-\eta(\mathcal{J} v, u, w)+\eta(w, u, \mathcal{J} v) \\
& -\eta(v, u, \mathcal{J} w)+\eta(\mathcal{J} w, u, v)+\eta(u, v, \mathcal{J} w) .
\end{aligned}
$$

Taking in (25) cyclic permutations over $u, v, w$, using again the symmetries (22) of $\eta$ and that $N_{\mathcal{J}}(u, v, w)$ is completely skew we obtain (24).

The next lemma concludes the proof of Proposition 25 and Theorem 23.

Lemma 29. The torsion of $\tilde{D}$ satisfies relation (17).

Proof. From relations (20) and (7), we obtain

$$
\begin{aligned}
& T^{\tilde{D}}(u, v, w)-T^{D^{(1)}}(u, v, w)= \\
& -\frac{1}{8} \sum_{(u, v, w) \text { cyclic }}(\eta(\mathcal{J} v, u, w)+\eta(w, u, \mathcal{J} v)-\eta(v, u, \mathcal{J} w)-\eta(\mathcal{J} w, u, v)) \\
& =-\frac{1}{4} \sum_{(u, v, w) \text { cyclic }}(\eta(\mathcal{J} u, w, v)+\eta(u, v, \mathcal{J} w)),
\end{aligned}
$$

where in the last equality we have used the symmetries (22) of $\eta$. From (23) we then obtain

$$
T^{\tilde{D}}(u, v, w)=\frac{1}{4} \sum_{(u, v, w) \text { cyclic }}(\eta(u, v, \mathcal{J} w)+\eta(\mathcal{J} u, v, w)),
$$

which implies (17), from Lemma 28,

\section{Generalized almost hypercomplex structures}

\subsection{Intrinsic torsion of a generalized almost hypercom- plex structure}

Let $\left(\mathcal{J}_{1}, \mathcal{J}_{2}, \mathcal{J}_{3}\right)$ be a generalized almost hypercomplex structure on a Courant algebroid $E$ and $\partial_{\mathbb{H}}: E^{*} \otimes \Lambda_{\mathbb{H}}^{1,1} E^{*} \rightarrow \Lambda^{3} E^{*}$ the algebraic torsion map defined by (9), where

$\operatorname{ad}_{\left(\mathcal{J}_{i}\right)}(E)=\Lambda_{\mathbb{H}}^{1,1} E^{*}:=\left\{\alpha \in \Lambda^{2} E^{*}, \alpha\left(\mathcal{J}_{i} u, \mathcal{J}_{i} v\right)=\alpha(u, v), u, v \in E, i=1,2,3\right\}$. 
Lemma 30. The endomorphism $P \in \Gamma\left(\operatorname{End}\left(\Lambda^{3} E^{*}\right)\right)$ defined by

$$
P:=\frac{2}{3} \sum_{i=1}^{3} \Pi_{\mathcal{J}_{i}}
$$

is a projector with $\operatorname{ker} P=\operatorname{im} \partial_{\mathbb{H}}$. In particular, $\operatorname{im} P$ is a complement of $\operatorname{im} \partial_{\mathbb{H}}$ in $\Lambda^{3} E^{*}$.

Proof. For any generalized almost complex structure $\mathcal{J}$, define the endomorphism $\Pi_{\mathcal{J}}^{0,2}$ of $\Lambda^{2} E^{*} \otimes E$ by $\left\langle\left(\Pi_{\mathcal{J}}^{0,2} \alpha\right)(u, v), w\right\rangle=\left(\Pi_{\mathcal{J}} \alpha\right)(u, v, w)$, where $\alpha \in \Lambda^{2} E^{*} \otimes E^{*}, u, v, w \in E$ and $\left(\Pi_{\mathcal{J}} \alpha\right)(u, v, w)$ is given by (10). Then $\Pi_{\mathcal{J}}^{0,2}$ coincides with the operator defined by relation (5) of [14]. We obtain that $(P \alpha)(u, v, w)=\langle p(\alpha)(u, v), w\rangle$ where $p=\frac{2}{3} \sum_{i=1}^{3} \Pi_{\mathcal{J}_{i}}^{0,2} \in \Gamma\left(\operatorname{End}\left(\Lambda^{2} E^{*} \otimes E\right)\right)$ is the map from Lemma 1 of [14]. As $p$ is a projector, $P$ is also a projector. From Lemma 20 ii), im $\partial_{\mathbb{H}} \subset \operatorname{ker} P$. Let

$$
\tilde{\pi}: \Lambda^{3} E^{*} \rightarrow E^{*} \otimes \Lambda_{\mathbb{H}}^{1,1} E^{*},(\tilde{\pi} \alpha)(u, v, w):=\alpha(u, v, w)+\sum_{i=1}^{3} \alpha\left(u, \mathcal{J}_{i} v, \mathcal{J}_{i} w\right)
$$

It is straightforward to check that

$$
\partial_{\mathbb{H}} \circ \tilde{\pi}=6\left(\operatorname{Id}_{\Lambda^{3} E^{*}}-P\right),
$$

which implies ker $P \subset \operatorname{im} \partial_{\mathbb{H}}$ and thus im $\partial_{\mathbb{H}}=\operatorname{ker} P$. As $P$ is a projector, $\operatorname{im} \partial_{\mathbb{H}}=\operatorname{ker} P$ is a complement of im $P$ in $\Lambda^{3} E^{*}$.

As in the previous section, we will identify $\left(\Lambda^{3} E^{*}\right) /\left(\operatorname{im} \partial_{\mathbb{H}}\right)$ with $\operatorname{im} P$ and consider the intrinsic torsion $t_{\left(\mathcal{J}_{i}\right)}$ of $\left(\mathcal{J}_{1}, \mathcal{J}_{2}, \mathcal{J}_{3}\right)$ as a section of im $P$.

Corollary 31. The intrinsic torsion $t_{\left(\mathcal{J}_{i}\right)}$ of $\left(\mathcal{J}_{1}, \mathcal{J}_{2}, \mathcal{J}_{3}\right)$ is given by $\frac{1}{6} \sum_{i=1}^{3} N_{\mathcal{J}_{i}}$.

Proof. Let $D$ be a hypercomplex connection. By Corollary 22 we have that $\Pi_{\mathcal{J}_{i}}\left(T^{D}\right)=\frac{1}{4} N_{\mathcal{J}_{i}}$. So $t_{\left(\mathcal{J}_{i}\right)}=P\left(T^{D}\right)=\frac{1}{6} \sum_{i=1}^{3} N_{\mathcal{J}_{i}}$.

\subsection{Integrability using torsion-free generalized connec- tions}

Our aim in this section is to prove the next theorem.

Theorem 32. A generalized almost hypercomplex structure $\left(\mathcal{J}_{1}, \mathcal{J}_{2}, \mathcal{J}_{3}\right)$ on a Courant algebroid $E$ is integrable if and only if there is a torsion-free generalized connection $D$ such that $D \mathcal{J}_{i}=0$ for all $i=1,2,3$. 
Part of the statement of Theorem 32 is obvious: if there is a torsion-free generalized connection which preserves all $\mathcal{J}_{i}$, then $\mathcal{J}_{i}$ are integrable from Theorem 23. The converse statement is proved in the next proposition.

Proposition 33. Let $\left(\mathcal{J}_{1}, \mathcal{J}_{2}, \mathcal{J}_{3}\right)$ be a generalized almost hypercomplex structure and $D$ a generalized connection on $E$, such that $D \mathcal{J}_{1}=0$. Define

$$
D^{(1)}:=D-\frac{1}{2} \mathcal{J}_{2} D \mathcal{J}_{2}
$$

and

$$
\tilde{D}:=D^{(1)}-\frac{1}{6} \tilde{\pi}\left(T^{D^{(1)}}\right),
$$

where $\tilde{\pi}$ is the map (28). Then $D^{(1)}$ and $\tilde{D}$ are generalized hypercomplex connections. Moreover,

$$
T^{\tilde{D}}(u, v, w)=\frac{1}{6} \sum_{i=1}^{3} N_{\mathcal{J}_{i}}(u, v, w) .
$$

In particular, if $\left(\mathcal{J}_{1}, \mathcal{J}_{2}, \mathcal{J}_{3}\right)$ is a generalized hypercomplex structure, then $\tilde{D}$ is torsion-free (and hypercomplex).

Proof. The existence of a generalized connection $D$ with $D \mathcal{J}_{1}=0$ follows from the proof of Lemma 26 (take any generalized connection, say $D^{(0)}$, and define $\left.D:=D^{(0)}-\frac{1}{2} \mathcal{J}_{1} D^{(0)} \mathcal{J}_{1}\right)$. The same argument shows that $D^{(1)}$ is a generalized connection which preserves $\mathcal{J}_{2}$. As $D \mathcal{J}_{1}=0$ and $\mathcal{J}_{2}$ anticommutes with $\mathcal{J}_{1}$, we obtain that $D_{u} \mathcal{J}_{2}$ anti-commutes with $\mathcal{J}_{1}$ as well, for any $u \in E$. Then

$$
D_{u}^{(1)} \mathcal{J}_{1}=D_{u} \mathcal{J}_{1}-\frac{1}{2}\left[\mathcal{J}_{2} D_{u} \mathcal{J}_{2}, \mathcal{J}_{1}\right]=-\frac{1}{2}\left[\mathcal{J}_{2} D_{u} \mathcal{J}_{2}, \mathcal{J}_{1}\right]=0
$$

As $D^{(1)} \mathcal{J}_{1}=D^{(1)} \mathcal{J}_{2}=0$ also $D^{(1)} \mathcal{J}_{3}=0$ and $D^{(1)}$ is hypercomplex. This proves the statement on $D^{(1)}$.

It remains to prove the statements on $\tilde{D}$. Let $\eta:=-\frac{1}{6} \tilde{\pi}\left(T^{D^{(1)}}\right)$. With this notation, $\tilde{D}=D^{(1)}+\eta$. Since $\operatorname{im} \tilde{\pi} \subset E^{*} \otimes \Lambda_{\mathbb{H}}^{1,1} E^{*}$ and $D^{(1)} \mathcal{J}_{i}=0$, also $\tilde{D} \mathcal{J}_{i}=0$ for all $i=1,2,3$, i.e. $\tilde{D}$ is hypercomplex. The torsion of $\tilde{D}$ is given by

$$
T^{\tilde{D}}=T^{D^{(1)}}-\frac{1}{6}\left(\partial_{\mathbb{H}} \circ \tilde{\pi}\right)\left(T^{D^{(1)}}\right)=P\left(T^{D^{(1)}}\right)=\frac{1}{6} \sum_{i=1}^{3} N_{\mathcal{J}_{i}},
$$

where in the second equality we used (29) and in the third equality we used (13) (which holds since $D^{(1)}$ is hypercomplex). 


\section{Generalized almost Hermitian structures: integrability and torsion-free generalized con- nections}

In this section we characterize the integrability of generalized almost Hermitian structures using Levi-Civita connections (see Theorem 36 below). We begin with the following simple lemma.

Lemma 34. Let $(G, \mathcal{J})$ be a generalized Kähler structure on E. Then

$$
[u, \mathcal{J} v]_{+}-\mathcal{J}[u, v]_{+}=0, \forall u \in \Gamma\left(E_{-}\right), v \in \Gamma\left(E_{+}\right) .
$$

Above we denoted by $e_{ \pm}$the $E_{ \pm}$-components of a vector $e \in E$ in the decomposition $E=E_{+} \oplus E_{-}$determined by $G$. Similarly,

$$
[u, \mathcal{J} v]_{-}-\mathcal{J}[u, v]_{-}=0, \forall u \in \Gamma\left(E_{+}\right), v \in \Gamma\left(E_{-}\right) .
$$

Proof. Relation (33) is equivalent to

$$
[u, v]_{+} \in \Gamma\left(\left(E_{+}\right)_{\mathbb{C}} \cap L_{1}\right), \forall u \in \Gamma\left(\left(E_{-}\right)_{\mathbb{C}}\right), v \in \Gamma\left(\left(E_{+}\right)_{\mathbb{C}} \cap L_{1}\right),
$$

where we have denoted by $L_{1}$ the $(1,0)$-bundle of $\mathcal{J}_{1}=\mathcal{J}$. Remark that $\left(E_{+}\right)_{\mathbb{C}} \cap L_{1}=\left(E_{+}\right)_{\mathbb{C}} \cap L_{2}$, where $L_{2}$ is the $(1,0)$-bundle of $\mathcal{J}_{2}=G$ end $\mathcal{J}$ (since $\mathcal{J}_{1}=\mathcal{J}_{2}$ on $\left.E_{+}\right)$. In (35) we distinguish two cases: a) $u \in \Gamma\left(L_{1} \cap\left(E_{-}\right) \mathbb{C}\right)$; b) $u \in \Gamma\left(\bar{L}_{1} \cap\left(E_{-}\right)_{\mathbb{C}}\right)$. In case a), relation (35) follows from the integrability of $\mathcal{J}$. In case b), relation (35) follows from the integrability of $\mathcal{J}_{2}$.

Remark 35. When the Courant algebroid is exact the above lemma can be proved using the Bismut connection for generalized Kähler structures, constructed in [18]. More precisely, for a generalized Kähler structure $(G, \mathcal{J})$ on an exact Courant algebroid $E$, there is a unique generalized connection $D$ (called in [18 the Bismut connection), such that $D G=0, D \mathcal{J}=0$, and whose torsion is of type $(2,1)+(1,2)$ with respect to $\mathcal{J}$. The expression of $D$ is given in Theorem 3.1 of [18. Its mixed components $D_{u} v$ and $D_{v} u$, for $u \in \Gamma\left(E_{+}\right)$and $v \in \Gamma\left(E_{-}\right)$, are $D_{u} v:=[u, v]_{-}$and $D_{v} u=[v, u]_{+}$. Relation (73) follows from $D \mathcal{J}=0$.

Theorem 36. A generalized almost Hermitian structure $(G, \mathcal{J})$ on a Courant algebroid $E$ is generalized Kähler if and only if there is a Levi-Civita connection of $G$ which preserves $\mathcal{J}$.

Proof. In one direction the statement is obvious: if there is a Levi-Civita connection $D$ of $G$ which preserves $\mathcal{J}$, then it preserves also $\mathcal{J}_{2}=G^{\text {end }} \mathcal{J}$ and we deduce that both $\mathcal{J}$ and $\mathcal{J}_{2}$ are integrable (from Theorem [23, because $D$ is torsion free). We obtain that $(G, \mathcal{J})$ is generalized Kähler. The converse statement follows from the next theorem. 
Theorem 37. Let $(G, \mathcal{J})$ be a generalized Kähler structure on a Courant algebroid $E$ and $D$ a Levi-Civita connection. Then the generalized connection

$$
\tilde{D}=D-\frac{1}{2} \mathcal{J} D \mathcal{J}-\frac{1}{4}\left\{A^{\mathrm{sym}}, \mathcal{J}\right\}
$$

is a torsion-free generalized connection compatible with $(G, \mathcal{J})$.

Proof. From Proposition 25 we know that $\tilde{D}$ is torsion-free and complex $(\tilde{D} \mathcal{J}=0)$. So it suffices to show that $\tilde{D} G=0$. Since $D G=0$ and $\mathcal{J}$ is $G$ skew-symmetric, the anticommuting endomorphisms $\mathcal{J}$ and $D_{u} \mathcal{J}(u \in E)$ are both $G$-skew and, hence, $\mathcal{J} D_{u} \mathcal{J}$ as well. We conclude that the generalized connection $D-\frac{1}{2} \mathcal{J} D \mathcal{J}$ preserves $G$.

It remains to check that $\left\{A_{u}^{\mathrm{sym}}, \mathcal{J}\right\}$ is $G$-skew-symmetric for all $u \in E$. We know that it is skew-symmetric with respect to $\langle\cdot, \cdot\rangle$, since both $D-$ $\frac{1}{2} \mathcal{J} D \mathcal{J}$ and $\tilde{D}$ are generalized connections. Therefore it suffices to check that $\left\{A_{u}^{\mathrm{sym}}, \mathcal{J}\right\}$ preserves the decomposition $E=E_{+} \oplus E_{-}$. Since $\mathcal{J}$ does, we only need to check that $\left\langle A_{u}^{\mathrm{sym}} E_{+}, E_{-}\right\rangle=0$. We check that $\left\langle A_{u} E_{ \pm}, E_{\mp}\right\rangle=0$, which implies the latter. Let $v \in \Gamma\left(E_{ \pm}\right), w \in \Gamma\left(E_{\mp}\right)$. If $u \in E_{ \pm}$, then $\left\langle A_{u} v, w\right\rangle=\left\langle\left(D_{v} \mathcal{J}\right) u, w\right\rangle=0$, because $D_{v} \mathcal{J}$ preserves the decomposition $E=E_{+} \oplus E_{-}($for all $v \in E)$. For $u \in E_{\mp}$, we use a) that $D$ has zero torsion to express derivatives by brackets with the help of the previous equation $\left\langle\left(D_{E} \mathcal{J}\right) E_{ \pm}, E_{\mp}\right\rangle=0$ and the property that $D$ preserves the subbundles $E_{ \pm}$ and b) Lemma 34:

$$
\left\langle A_{u} v, w\right\rangle=\left\langle\left(D_{v} \mathcal{J}\right) u, w\right\rangle=\left\langle D_{v}(\mathcal{J} u)-\mathcal{J} D_{v} u, w\right\rangle \stackrel{a)}{=}\langle[v, \mathcal{J} u]-\mathcal{J}[v, u], w\rangle \stackrel{b)}{=} 0 .
$$

Note that the equations $\left\langle\left(D_{E_{ \pm}} \mathcal{J}\right) E, E_{\mp}\right\rangle=\left\langle\left(D_{E_{ \pm}} \mathcal{J}\right) E_{\mp}, E\right\rangle=0$, established in the proof, can be also written as:

$$
\left(D_{E_{ \pm}} \mathcal{J}\right) E_{\mp}=0, \quad\left(D_{E_{ \pm}} \mathcal{J}\right) E_{ \pm} \subset E_{ \pm}
$$

\section{Generalized almost hyper-Hermitian struc- tures: integrability and torsion-free gener- alized connections}

Theorem 38. A generalized almost hyper-Hermitian structure $\left(G, \mathcal{J}_{1}, \mathcal{J}_{2}, \mathcal{J}_{3}\right)$ on a Courant algebroid $E$ is generalized hyper-Kähler if and only if there is a Levi-Civita connection $D$ of $G$ which satisfies $D \mathcal{J}_{i}=0$, for all $i=1,2,3$. 
Proof. If there is a Levi-Civita connection $D$ of $G$ which is hypercomplex, then $\left(G, \mathcal{J}_{i}\right)$ is generalized Kähler (see Theorem 36 ).

For the converse statement, let $\left(G, \mathcal{J}_{1}, \mathcal{J}_{2}, \mathcal{J}_{3}\right)$ be a generalized hyperKähler structure and $D$ a generalized Levi-Civita connection of $G$ with $D \mathcal{J}_{1}=0$ (which exists, by Theorem 36). We will show that the generalized connection $\tilde{D}$ constructed in Proposition 33 , starting from $D$, is a Levi-Civita connection of $G$ which preserves the $\mathcal{J}_{i}$. Define the generalized connections

$$
D_{u}^{(1)}:=D_{u}-\frac{1}{2} \mathcal{J}_{2} D_{u} \mathcal{J}_{2}
$$

and

$$
\tilde{D}_{u}:=D_{u}^{(1)}+\eta_{u}=D_{u}-\frac{1}{2} \mathcal{J}_{2} D_{u} \mathcal{J}_{2}+\eta_{u},
$$

where $\eta:=-\frac{1}{6} \tilde{\pi}\left(T^{D^{(1)}}\right)$. From Proposition 33, $D^{(1)}$ and $\tilde{D}$ are hypercomplex and $\tilde{D}$ is torsion-free. We claim that $\tilde{D} G=0$, which proves the theorem.

Note first that $D^{(1)} G=0$, since $D G=0$ and $\mathcal{J}_{2} D_{u} \mathcal{J}_{2}$ is skew-symmetric with respect to $G$. So it suffices to show that $\eta(u, v, w)=0$ for all $u \in E$, $v \in E_{ \pm}, w \in E_{\mp}$. Note that $\eta=-\frac{1}{6} \tilde{\pi}\left(T^{D^{(1)}}\right)$ has the following expression:

$$
\begin{aligned}
& \eta(u, v, w)= \\
& \frac{1}{12}\left\langle\left(D_{\mathcal{J}_{1} v} \mathcal{J}_{2}\right)\left(\mathcal{J}_{3} w\right)+\left(D_{\mathcal{J}_{2} v} \mathcal{J}_{2}\right)(w)-\left(D_{\mathcal{J}_{3} v} \mathcal{J}_{2}\right)\left(\mathcal{J}_{1} w\right)+\mathcal{J}_{2}\left(D_{v} \mathcal{J}_{2}\right)(w), u\right\rangle \\
& -\frac{1}{12}\left\langle\mathcal{J}_{3}\left(D_{\mathcal{J}_{1} w} \mathcal{J}_{2}\right)(u)-\left(D_{\mathcal{J}_{2} w} \mathcal{J}_{2}\right)(u)-\mathcal{J}_{1}\left(D_{\mathcal{J}_{3} w} \mathcal{J}_{2}\right)(u)-\mathcal{J}_{2}\left(D_{w} \mathcal{J}_{2}\right)(u), v\right\rangle,
\end{aligned}
$$

for any $u, v, w \in \Gamma(E)$. For all $u \in E, v \in E_{ \pm}, w \in E_{\mp}$, each summand belongs either to the set $\left\langle\left(D_{E_{ \pm}} \mathcal{J}_{\alpha}\right) E_{\mp}, E\right\rangle$ or to $\left\langle\left(D_{E_{\mp}} \mathcal{J}_{\alpha}\right) E, E_{ \pm}\right\rangle$, which both reduce to zero by (36).

\section{Part II}

\section{The space of local Dirac generating opera- tors}

Let $E$ be an oriented Courant algebroid with anchor $\pi: E \rightarrow T M$, scalar product $\langle\cdot, \cdot\rangle$ and Dorfman bracket $[\cdot, \cdot]$. We assume that $\langle\cdot, \cdot\rangle$ is of neutral signature $(n, n)$. We denote by $\mathrm{Cl}(E)$ the bundle of Clifford algebras over 
$(E,\langle\cdot, \cdot\rangle)$ with the Clifford relation $e^{2}=\langle e, e\rangle, e \in E$. Let $S \rightarrow M$ be a real vector bundle of irreducible $\mathrm{Cl}(E)$-modules. We will call $S$ a spinor bundle over $\mathrm{Cl}(E)$. The representation of $\mathrm{Cl}(E)$ on $S$, denoted by

$$
\gamma: \mathrm{Cl}(E) \rightarrow \operatorname{End}(S), \quad a \mapsto \gamma(a):=\gamma_{a},
$$

is an isomorphism of algebra bundles. To simplify notation, we shall sometimes write as for the Clifford action $\gamma_{a} s$ of $a \in \mathrm{Cl}(E)$ on $s \in S$.

Recall that the Clifford algebra bundle $\mathrm{Cl}(E)$ is $\mathbb{Z}_{2}$-graded. We denote the subbundle of $\mathrm{Cl}(E)$ of degree $i \in \mathbb{Z}_{2}$ by $\mathrm{Cl}^{i}(E)$. Since $\langle\cdot, \cdot\rangle$ has neutral signature, the bundle $S$ has a compatible $\mathbb{Z}_{2}$-grading denoted by $S=S^{0} \oplus S^{1}$, where $S^{0}=\frac{1}{2} \gamma_{(1+\omega)} S$ and $S^{1}=\frac{1}{2} \gamma_{(1-\omega)} S$, with $\omega=e_{1} \wedge \cdots \wedge e_{2 n}$ the volume form of $E$, determined by a positive oriented orthonormal basis of $E$, and considered as an element of $\mathrm{Cl}(E)$ (see e.g. Proposition 3.6 of [21]). An argument analogous to the proof of Proposition 5.10 of 21] shows that the $\mathrm{Cl}^{0}(E)$-submodules $S^{0}$ and $S^{1}$ are pointwise inequivalent and irreducible. There is an induced $\mathbb{Z}_{2}$-grading on $\operatorname{End}(\Gamma(S))$ and, in particular, on the algebra of differential operators on $S$, which includes $\Gamma($ End $S) \subset \operatorname{End}(\Gamma(S)$ ) as the subalgebra of operators of 0 -th order. We will denote by

$$
[A, B]=A B-(-1)^{\operatorname{deg} A \operatorname{deg} B} B A
$$

the super commutator of two homogeneous elements $A, B \in \operatorname{End}(\Gamma(S))$, where $\operatorname{deg} A$ stands for the degree of $A$.

Definition 39. A first order odd differential operator $\not d$ on a spinor bundle $S$ over $\mathrm{Cl}(E)$ is called a Dirac generating operator for $E$ if for all $f \in C^{\infty}(M)$ and $e, e_{1}, e_{2} \in \Gamma(E)$,

i) $\left.\left[[\phi, f], \gamma_{e}\right]\right]=\pi(e)(f)$,

ii) $\left[\left[\phi, \gamma_{e_{1}}\right], \gamma_{e_{2}}\right]=\gamma_{\left[e_{1}, e_{2}\right]}$ and

iii) $\not^{2} \in C^{\infty}(M)$.

Note that given $(E,\langle\cdot, \cdot\rangle)$ and $\not$ one can reconstruct the full Courant algebroid structure from i) and ii). This is why the operator $\not d$ is called generating.

Proposition 40. Suppose that there is a Dirac generating operator $\not d$ for $E$ on $S$. Then the set of Dirac generating operators for $E$ on $S$ has the structure of an affine space modelled on the vector space

$$
V_{\not d}:=\left\{e \in \Gamma(E) \mid\left[\not d, \gamma_{e}\right] \in C^{\infty}(M)\right\} .
$$

In particular, $V_{d}$ is independent of the choice of Dirac generating operator d. 
Proof. We first check that $\not^{\prime}:=\not d+\gamma_{e_{0}}$ is a Dirac generating operator for all $e_{0} \in V_{\not d}$. Since $\left[\gamma_{e_{0}}, f\right]=0$ and $\left[\gamma_{e_{0}}, \gamma_{e_{1}}\right]=2\left\langle e_{0}, e_{1}\right\rangle \in C^{\infty}(M)$ the properties i) and ii) in Definition 39 for $\not d$ immediately imply the same properties for $\not d^{\prime}$. Finally, the equation $\left(\phi^{\prime}\right)^{2}=\not^{2}+\left[\not d, \gamma_{e_{0}}\right]+\left\langle e_{0}, e_{0}\right\rangle$ shows that property iii) holds for $\phi^{\prime}$ if it holds for $\not d$ and $e_{0} \in V_{\phi d}$.

Conversely, we show that given Dirac generating operators $\not d$ and $\phi^{\prime}$, there exists $e_{0} \in V_{\not d}$ such that $L:=\not d^{\prime}-\not d=\gamma_{e_{0}}$. We first observe that $[L, f]$ is a 0 -th order operator of odd degree for all $f \in C^{\infty}(M)$. By property i) it satisfies $\left[[L, f], \gamma_{e}\right]=0$ for all $e \in \Gamma(E)$. This implies that $[L, f]$ commutes with $\mathrm{Cl}^{0}(E)$. Being of odd degree, it interchanges $S^{0}$ and $S^{1}$ and we deduce that $[L, f]=0$ since the irreducible $\mathrm{Cl}^{0}(\mathrm{E})$-modules $S^{0}$ and $S^{1}$ are inequivalent. This shows that the odd differential operator $L$ is of 0 -th order, that is $L=\gamma_{a}$ for some $a \in \Gamma\left(\mathrm{Cl}^{1}(E)\right)$.

Next we consider the even 0 -the order operator

$$
L^{\prime}:=\left[L, \gamma_{e}\right]=\gamma(a e+e a),
$$

$e \in \Gamma(E)$. It commutes with $\mathrm{Cl}^{1}(E)$, in virtue of property ii), and is hence a scalar operator (since $\langle\cdot, \cdot\rangle$ has neutral signature). We conclude that $a e+e a$ is a scalar in $\mathrm{Cl}(E)$, for any $e \in E$. This easily implies that $a=: e_{0} \in \Gamma(E)$, by a straightforward computation in which $e$ runs through the elements of an orthonormal frame. Now property iii) implies that $e_{0} \in V_{d d}$.

The last claim is now obvious: if $\not d$ and $\phi^{\prime}$ are two Dirac generating operators then $\not d^{\prime}=\not d+\gamma_{e}$ for $e \in V_{\not d} \subset \Gamma(E)$ which implies $V_{\not^{\prime}}=V_{\not d}$.

The next theorem is our main result in this section.

Theorem 41 (Alekseev-Xu). Let $E$ be a regular Courant algebroid with scalar product of neutral signature. Every spinor bundle $S$ over $\mathrm{Cl}(E)$ admits locally a Dirac generating operator.

We divide the proof of Theorem 41 into several steps. Let $D$ be a generalized connection on $E$. The existence of $D$ is ensured by Example 5 , The generalized connection $D$ induces an $E$-connection in $\mathrm{Cl}(E)$, which we denote again by $D$. Next we choose an $E$-connection $D^{S}$ on $S$ compatible with $D$ in the sense that

$$
D_{e}^{S}(a s)=\left(D_{e} a\right) s+a D_{e}^{S} s
$$

for all $e \in \Gamma(E), a \in \Gamma(\mathrm{Cl}(E)), s \in \Gamma(S)$.

The existence of such a connection $D^{S}$ can be shown as follows. The bundle $(E,\langle\cdot, \cdot\rangle)$ admits locally a spin structure. To this structure we can associate a spinor bundle $\Sigma$ over some domain $U \subset M$. The $E$-connection $D$ on $E$ induces an $E$-connection $D^{\Sigma}$ on $\Sigma$. The connection form of $D^{\Sigma}$ with 
respect to a local trivialization of the spin structure is one half of the connection form of $D$ with respect to the corresponding local orthonormal frame of $E$. (Both forms can be considered as local sections of $E^{*} \otimes \mathfrak{s o}(E)$, after identifying $\mathfrak{s p i n}(E) \cong \mathfrak{s o}(E)$ via the adjoint representation ad $: \mathfrak{s p i n}(E) \rightarrow \mathfrak{s o}(E)$, $\operatorname{ad}_{u} v:=u v-v u$.) In more concrete terms, let $\left(e_{i}\right)$ be an orthonormal frame of $\left.E\right|_{U}$ and $\left(\sigma_{\alpha}\right)$ a frame of $\Sigma$ such that

$$
e_{i} \sigma_{\alpha}=\sum_{\beta} C_{i \alpha}^{\beta} \sigma_{\beta}
$$

where $C_{i \alpha}^{\beta}$ are constants. Let $\left(\omega_{i j}\right)$ be the (skew-symmetric) matrix of 1-forms defined by

$$
\begin{aligned}
D_{v}\left(e_{k}\right) & =-\epsilon_{k} \sum_{j} \omega_{j k}(v) e_{j}=2 \sum_{j<p} \omega_{p j}(v)\left(\epsilon_{p} e_{p}^{*} \otimes e_{j}-\epsilon_{j} e_{j}^{*} \otimes e_{p}\right)\left(e_{k}\right) \\
& =2 \sum_{j<p} \omega_{p j}(v)\left(e_{p} \wedge e_{j}\right)\left(e_{k}\right),
\end{aligned}
$$

where $\epsilon_{k}=\left\langle e_{k}, e_{k}\right\rangle \in\{ \pm 1\}$. Then $D_{e}^{\Sigma}\left(\sigma_{\alpha}\right):=\frac{1}{2} \sum_{i<j} \omega_{j i}(v) e_{i} e_{j} \sigma_{\alpha}$ is compatible with $\left.D\right|_{U}$. (Note that the element $\frac{1}{2} e_{i} e_{j} \in \mathfrak{s p i n}(E) \subset \mathrm{Cl}(\mathrm{E})(i \neq j)$ acts under the adjoint representation as $\epsilon_{j} e_{j}^{*} \otimes e_{i}-\epsilon_{i} e_{i}^{*} \otimes e_{j} \in \mathfrak{s o}(E)$ and the latter corresponds to the bivector $e_{j} \wedge e_{i} \in \Lambda^{2} E$.) Since $(E,\langle\cdot, \cdot\rangle)$ has neutral signature, $\left.S\right|_{U}$ and $\Sigma$ differ only by a real line bundle $L$ over $U:\left.S\right|_{U} \cong \Sigma \otimes L$. Choosing an $E$-connection in $L$, we obtain an $E$-connection $D^{S, U}$ in $S_{U}$ by taking the tensor product with the connection $D^{\Sigma}$. By considering an open covering $\left(U_{i}\right)$ of $M$ and a corresponding partition of unity, we can glue the $E$-connections $D^{S, U_{i}}$ to an $E$-connection $D^{S}$.

The $E$-connection $D^{S}$ gives rise to a first order differential operator on $S$, which we call the Dirac operator:

$$
\not D^{S}=\frac{1}{2} \sum_{i=1}^{2 n} \tilde{e}_{i} D_{e_{i}}^{S},
$$

where $\left(e_{i}\right)$ is any local frame of $E$ and $\left(\tilde{e}_{i}\right)$ is the metrically dual frame, that is $\left\langle e_{i}, \tilde{e}_{j}\right\rangle=\delta_{i j}$.

Lemma 42. For any generalized connection $D$ and compatible E-connection $D^{S}$, the operator

$$
\not d=\not D^{S}+\frac{1}{4} \gamma_{T}
$$

satisfies conditions i) and ii) from Definition 39. Above $T \in \Gamma\left(\wedge^{3} E^{*}\right) \cong$ $\Gamma\left(\wedge^{3} E\right) \subset \Gamma(\mathrm{Cl}(E))$ denotes the torsion of $D$. 
Proof. We compute $\left.\left[[\not d, f], \gamma_{v}\right]\right]=\left[\left[\not D^{S}, f\right], \gamma_{v}\right]$ for $f \in C^{\infty}(M)$ and $v \in \Gamma(E)$. We find

$$
\left[\not D^{S}, f\right]=\frac{1}{2} \sum_{i} \pi\left(e_{i}\right)(f) \gamma_{\tilde{e}_{i}}, \quad\left[\left[\not D^{S}, f\right], \gamma_{v}\right]=\sum_{i} \pi\left(e_{i}\right)(f)\left\langle\tilde{e}_{i}, v\right\rangle=\pi(v)(f) .
$$

This shows that i) in Definition 39 is satisfied.

Next we compute

$$
\left[\not D^{S}, \gamma_{v}\right]=\frac{1}{2} \sum_{i} \gamma_{\tilde{e}_{i}} \gamma_{D_{e_{i}} v}+D_{v}^{S}, \quad\left[\left[\not D^{S}, \gamma_{v}\right], \gamma_{w}\right]=\frac{1}{2} \sum_{i}\left[\gamma_{\tilde{e}_{i}} \gamma_{D_{e_{i}} v}, \gamma_{w}\right]+\gamma_{D_{v} w}
$$

where $w \in \Gamma(E)$. A simple calculation in the Clifford algebra shows that for all $u, v, w \in E$ :

$$
u v w-w u v=-2\langle u, w\rangle v+2\langle v, w\rangle u .
$$

So

$\frac{1}{2} \sum_{i}\left[\gamma_{\tilde{e}_{i}} \gamma_{D_{e_{i}} v}, \gamma_{w}\right]=-\sum_{i}\left\langle\tilde{e}_{i}, w\right\rangle \gamma_{D_{e_{i}} v}+\sum_{i}\left\langle D_{e_{i}} v, w\right\rangle \gamma_{\tilde{e}_{i}}=-\gamma_{D_{w} v}+\sum_{i}\left\langle D_{e_{i}} v, w\right\rangle \gamma_{\tilde{e}_{i}}$

and using that $D$ has torsion $T$ we obtain

$$
\begin{aligned}
{\left[\left[D^{S}, \gamma_{v}\right], \gamma_{w}\right] } & =\gamma_{D_{v} w-D_{w} v}+\sum_{i}\left\langle D_{e_{i}} v, w\right\rangle \gamma_{\tilde{e}_{i}} \\
& =\gamma_{T(v, w)}+\gamma_{[v, w]}-\gamma_{(D v)^{*} w}+\sum_{i}\left\langle D_{e_{i}} v, w\right\rangle \gamma_{\tilde{e}_{i}} \\
& =\gamma_{T(v, w)}+\gamma_{[v, w]} .
\end{aligned}
$$

A simple calculation in the Clifford algebra shows that (for any 3-form $T$ )

$$
\left[\left[\gamma_{T}, \gamma_{v}\right], \gamma_{w}\right]=-4 \gamma_{T(v, w)}
$$

Thus we can conclude that

$$
\left[\left[d, \gamma_{v}\right], \gamma_{w}\right]=\left[\left[\not D^{S}+\frac{1}{4} \gamma_{T}, \gamma_{v}\right], \gamma_{w}\right]=\gamma_{[v, w]} .
$$

So ii) in Definition 39 is also satisfied.

In order to conclude the proof of Theorem 41 we therefore need to find a locally defined generalized connection $D$ on $E$ and a compatible $E$-connection $D^{S}$ on $S$ such that condition iii) from Definition 39 holds as well. To analyze condition iii) in Definition 39 we will use the following lemma, see [7]. (When $\langle\cdot, \cdot\rangle$ has arbitrary signature, the next lemma still holds with the only difference that the restriction of $\langle\cdot, \cdot\rangle$ to $\mathcal{G}$ does not have neutral signature). 
Lemma 43. ([7) Let $E$ be a regular Courant algebroid with scalar product $\langle\cdot, \cdot\rangle$ of neutral signature and anchor $\pi: E \rightarrow T M$.

i) The bundle $\operatorname{ker} \pi \subset E$ is a coisotropic subbundle of $E$, that is $(\operatorname{ker} \pi)^{\perp} \subset$ $\operatorname{ker} \pi$.

ii) The bundle $E$ decomposes as $E=\operatorname{ker} \pi \oplus \mathcal{F}$ where $\mathcal{F}$ is isotropic.

iii) The bundle $\operatorname{ker} \pi$ decomposes as $\operatorname{ker} \pi=(\operatorname{ker} \pi)^{\perp} \oplus \mathcal{G}$ where $\mathcal{G}$ is orthogonal to $\mathcal{F}$.

iv) The decomposition $E=\left((\operatorname{ker} \pi)^{\perp} \oplus \mathcal{F}\right) \oplus \mathcal{G}$ is orthogonal with respect to $\langle\cdot, \cdot\rangle$. The restrictions of $\langle\cdot, \cdot\rangle$ to the two factors $(\operatorname{ker} \pi)^{\perp} \oplus \mathcal{F}$ and $\mathcal{G}$ have neutral signature.

The above lemma implies that for any $U \subset M$ sufficiently small, the bundle $\left.E\right|_{U}$ admits a frame $\left(p_{a}, q_{a}\right), a=1, \ldots, n$, such that $\left(p_{a}\right), a=1, \ldots, n$, span a maximally isotropic subbundle $\mathcal{P}$ of $\operatorname{ker} \pi,\left(p_{a}\right), a=1, \ldots, s$, span $(\operatorname{ker} \pi)^{\perp},\left(q_{a}\right), a=1, \ldots, n$, span a maximally isotropic subbundle $\mathcal{Q}$ of $E$, $q_{a} \in \operatorname{ker} \pi$ for any $a \geq s+1,\left\langle p_{a}, q_{b}\right\rangle=\delta_{a b}$ and $\left[\pi\left(q_{a}\right), \pi\left(q_{b}\right)\right]=0$ for any $a$, $b$. For the latter condition we are using that the image of $\pi$ is an integrable distribution on $M$ (by the axiom C2) in Definition 11). More precisely, using Lemma 43 iv), this basis can be constructed in the following way: start with any basis $q_{a}, a=1, \cdots, s$, of $\mathcal{F}$ such that $\left[\pi\left(q_{a}\right), \pi\left(q_{b}\right)\right]=0$ for any $a$, $b$. Consider the basis $p_{a}, a=1, \cdots, s$, of $(\operatorname{ker} \pi)^{\perp}$ such that $\left\langle p_{a}, q_{b}\right\rangle=\delta_{a b}$ for any $a, b$. Finally, choose a basis $p_{a}, q_{a}, a=s+1, \cdots, n$, of $\mathcal{G}$ such that $\left\langle p_{a}, p_{b}\right\rangle=\left\langle q_{a}, q_{b}\right\rangle=0$ and $\left\langle p_{a}, q_{b}\right\rangle=\delta_{a b}$ for any $a, b=s+1, \cdots, n$. The following inclusions summarize the properties of the two complementary maximally isotropic subbundles $\mathcal{P}$ and $\mathcal{Q}$ of $E$ :

$$
(\operatorname{ker} \pi)^{\perp} \subset \mathcal{P} \subset \operatorname{ker} \pi, \quad \mathcal{F} \subset \mathcal{Q} \subset \mathcal{F}^{\perp}=\mathcal{F} \oplus \mathcal{G} .
$$

The next corollary will be useful in the proof of Lemma 45 below.

Corollary 44. For any $\sigma \in \Gamma(\operatorname{ker} \pi), \sum_{a=1}^{n} \pi\left(q_{a}\right)\left\langle\sigma, p_{a}\right\rangle=0$.

Proof. Each term in the above sum vanishes: if $a \leq s$ then $p_{a} \in \Gamma\left((\operatorname{ker} \pi)^{\perp}\right)$ and $\left\langle\sigma, p_{a}\right\rangle=0$. If $a \geq s+1$, then $q_{a} \in \Gamma(\operatorname{ker} \pi)$ and $\pi\left(q_{a}\right)=0$.

Let $\nabla$ be the connection on $\left.E\right|_{U}$ such that the frame $\left(p_{a}, q_{a}\right)$ is parallel. Then $\nabla$ is flat, preserves the scalar product $\langle\cdot, \cdot\rangle$ of $E$ and $\left.S\right|_{U}$ admits a flat connection $\nabla^{S}$ compatible with $\nabla$. Then $\nabla$ induces a generalized connection $D$ on $\left.E\right|_{U}$ and $\nabla^{S}$ induces an $E$-connection $D^{S}$ on $\left.S\right|_{U}$ which is compatible with $D$.

The next lemma concludes the proof of Theorem 41 .

Lemma 45. The operator (42) constructed using $D$ and $D^{S}$ satisfies $\not^{2} \in$ $C^{\infty}(U)$ and is a Dirac generating operator. 
Proof. The Dirac operator $\not D^{S}$ has the expression

$$
\not D^{S}=\frac{1}{2} \sum_{a}\left(p_{a} D_{q_{a}}^{S}+q_{a} D_{p_{a}}^{S}\right)=\frac{1}{2} \sum_{a} p_{a} D_{q_{a}}^{S},
$$

since $\pi\left(p_{a}\right)=0$. To see this it is sufficient to remark that the frame $\left(\tilde{q}_{a}, \tilde{p}_{b}\right)$ dual to the frame $\left(q_{a}, p_{b}\right)$ is precisely $\left(p_{a}, q_{b}\right)$. Its square is given by

$$
\left(\not D^{S}\right)^{2}=\frac{1}{4} \sum_{a} p_{a} p_{b} D_{q_{a}}^{S} D_{q_{b}}^{S}=\frac{1}{4} \sum_{a}\left\langle p_{a}, p_{b}\right\rangle \nabla_{\pi\left(q_{a}\right)}^{S} \nabla_{\pi\left(q_{b}\right)}^{S}=0
$$

where we used $\nabla^{S} p_{a}=0$, the flatness of $\nabla^{S}$ and $\left[\pi\left(q_{a}\right), \pi\left(q_{b}\right)\right]=0$ for any $a$, $b$.

Next, we compute $\not D^{S} \gamma_{T}+\gamma_{T} \not D^{S}=\left[\not D^{S}, \gamma_{T}\right]$. We write the torsion $T$ of $D$ as $T=\frac{1}{6} \sum T^{i j k} e_{i j k} \in \mathrm{Cl}(E)$, where $\left(e_{i}\right)$ is a $D$-parallel orthonormal frame and $e_{i j k}:=e_{i} e_{j} e_{k}$. The coefficients $T^{i j k}$ are given by

$$
T^{i j k}=T\left(\tilde{e}_{i}, \tilde{e}_{j}, \tilde{e}_{k}\right)=\epsilon_{i} \epsilon_{j} \epsilon_{k} T\left(e_{i}, e_{j}, e_{k}\right)=-\epsilon_{i} \epsilon_{j} \epsilon_{k}\left\langle\left[e_{i}, e_{j}\right], e_{k}\right\rangle,
$$

where $\left(\tilde{e}_{i}\right)$ is the frame of $\left.E\right|_{U}$ metrically dual to $\left(e_{i}\right)$, i.e. $\tilde{e}_{i}=\epsilon_{i} e_{i}$ with $\epsilon_{i}=\left\langle e_{i}, e_{i}\right\rangle$. Using the abbreviation $\gamma_{e_{i} e_{j} e_{k}}=\gamma_{i j k}$, we write

$$
\begin{aligned}
& 12\left[\not D^{S}, \gamma_{T}\right]=\sum_{i, j, k, \ell}\left[\gamma_{\tilde{e}_{\ell}} D_{e_{\ell}}^{S}, T^{i j k} \gamma_{i j k}\right] \\
& =\sum_{i, j, k, \ell} \pi\left(e_{\ell}\right)\left(T^{i j k}\right) \gamma_{\tilde{e}_{\ell}} \gamma_{i j k}+\sum_{i, j, k, \ell} T^{i j k} \gamma_{\tilde{e}_{\ell}} \gamma(\underbrace{D_{e_{\ell} e_{i j k}}}_{=0})+\sum_{i, j, k, \ell} T^{i j k}\left[\gamma_{\tilde{e}_{\ell}}, \gamma_{i j k}\right] D_{e_{\ell}}^{S} .
\end{aligned}
$$

Note that, for any fixed $\ell$,

$$
\sum_{i, j, k} T^{i j k}\left[\gamma_{\tilde{e}_{\ell}}, \gamma_{i j k}\right]=6 \sum_{j, k} T^{\ell j k} \gamma_{j k}
$$

Hence

$$
\sum_{i, j, k, \ell} T^{i j k}\left[\gamma_{\tilde{e}_{\ell}}, \gamma_{i j k}\right] D_{e_{\ell}}^{S}=-6 \sum_{j, k} \epsilon_{j} \epsilon_{k} \gamma_{j k} D_{\left[e_{j}, e_{k}\right]}^{S}
$$

To compute the last term we choose the orthonormal frame $\left(e_{i}\right)$ to be

$$
\left(e_{i}\right)_{i=1, \ldots, 2 n}=\left(\frac{1}{\sqrt{2}}\left(p_{a}+q_{a}\right), \frac{1}{\sqrt{2}}\left(p_{a}-q_{a}\right)\right)_{a=1, \ldots, n},
$$

where $\left(p_{a}, q_{a}\right)$ is the frame constructed above. Then $\pi\left[e_{j}, e_{k}\right]=\left[\pi e_{j}, \pi e_{k}\right]=0$, because $\left[\pi\left(q_{a}\right), \pi\left(q_{b}\right)\right]=0$. This implies that $D_{\left[e_{j}, e_{k}\right]}^{S}=0$, showing that

$$
\left[\not D^{S}, \gamma_{T}\right]=\frac{1}{12} \sum_{i, j, k, \ell} \pi\left(e_{\ell}\right)\left(T^{i j k}\right) \gamma_{\tilde{e}_{\ell}} \gamma_{i j k}
$$


From (43) and (45), we obtain

$$
\not d^{2}=\frac{1}{4}\left[\not D^{S}, \gamma_{T}\right]+\frac{1}{16} \gamma_{T}^{2}=\frac{1}{16}\left(\frac{1}{3} \sum_{i, j, k, \ell} \pi\left(e_{\ell}\right)\left(T^{i j k}\right) \gamma_{\tilde{e} \ell} \gamma_{i j k}+\gamma_{T}^{2}\right) .
$$

We compute

$\gamma_{T}^{2}=\frac{1}{4} \sum_{i, j, \ell, m, n} \epsilon_{\ell} T^{\ell i j} T^{\ell m n} \gamma_{i j m n}=\frac{1}{4} \sum_{i, j, \ell, m, n}^{\prime} \epsilon_{\ell} T^{\ell i j} T^{\ell m n} \gamma_{i j m n}-\sum_{i, j, r} \epsilon_{i} \epsilon_{j} \epsilon_{r}\left(T^{i j r}\right)^{2}$,

where the primed sum is only over pairwise distinct indices. Similarly,

$$
\sum_{i, j, k, \ell} \pi\left(e_{\ell}\right)\left(T^{i j k}\right) \gamma_{\tilde{e}_{\ell}} \gamma_{i j k}=\sum_{i, j, k, \ell}^{\prime} \pi\left(e_{\ell}\right)\left(T^{i j k}\right) \gamma_{\tilde{e}_{\ell}} \gamma_{i j k}+3 \sum_{j, k, \ell} \pi\left(e_{\ell}\right)\left(T^{\ell j k}\right) \gamma_{j k} .
$$

On the other hand, for any $j$ and $k$ fixed,

$$
\begin{aligned}
\sum_{\ell} \pi\left(e_{\ell}\right)\left(T^{\ell j k}\right) & =-\epsilon_{j} \epsilon_{k} \sum_{\ell} \pi\left(e_{\ell}\right)\left\langle\left[e_{j}, e_{k}\right], e_{\ell}\right\rangle \epsilon_{\ell} \\
& =-\epsilon_{j} \epsilon_{k} \sum_{a} \pi\left(q_{a}\right)\left\langle\left[e_{j}, e_{k}\right], p_{a}\right\rangle=0,
\end{aligned}
$$

where we used (44) and Corollary 44 (with $\sigma=\left[e_{j}, e_{k}\right]$, which is a section of $\operatorname{ker} \pi$ ). Combining the above relations we obtain

$$
\begin{aligned}
\not d^{2} & =\frac{1}{16}\left(\frac{1}{3} \sum_{i, j, k, \ell}^{\prime} \pi\left(e_{\ell}\right)\left(T^{i j k}\right) \gamma_{\tilde{e}_{\ell}} \gamma_{i j k}+\frac{1}{4} \sum_{i, j, \ell, m, n}^{\prime} \epsilon_{\ell} T^{\ell i j} T^{\ell m n} \gamma_{i j m n}\right) \\
& -\frac{1}{16} \sum_{i, j, r} \epsilon_{i} \epsilon_{j} \epsilon_{r}\left(T^{i j r}\right)^{2}
\end{aligned}
$$

We aim to prove that

$$
\not d^{2}=-\frac{1}{16} \sum_{i, j, k} \epsilon_{i} \epsilon_{j} \epsilon_{k}\left(T^{i j k}\right)^{2}
$$

For this, we need to show that

$$
\frac{1}{3} \sum_{i, j, k, \ell}^{\prime} \epsilon_{\ell} \pi\left(e_{\ell}\right)\left(T^{i j k}\right) \gamma_{\ell i j k}+\frac{1}{4} \sum_{i, j, \ell, m, n}^{\prime} \epsilon_{\ell} T^{\ell i j} T^{\ell m n} \gamma_{i j m n}=0 .
$$

To prove (48) we use axiom C1) of Definition 1, where indices of tensor components are metrically raised and lowered according to standard conventions: 
for any $i, j, k$ fixed,

$$
\begin{aligned}
0= & {\left[e_{i},\left[e_{j}, e_{k}\right]\right]-\left[\left[e_{i}, e_{j}\right], e_{k}\right]-\left[e_{j},\left[e_{i}, e_{k}\right]\right] } \\
= & \sum_{\ell}\left(-\left[e_{i}, T_{j k}{ }^{\ell} e_{\ell}\right]+\left[T_{i j}{ }^{\ell} e_{\ell}, e_{k}\right]+\left[e_{j}, T_{i k}{ }^{\ell} e_{\ell}\right]\right) \\
= & \sum_{\ell}\left(-\pi\left(e_{i}\right)\left(T_{j k}^{\ell}\right)+\pi\left(e_{j}\right)\left(T_{i k}^{\ell}\right)\right) e_{\ell}+\sum_{\ell, m}\left(T_{j k}{ }^{\ell} T_{i \ell}{ }^{m}-T_{i k}{ }^{\ell} T_{j \ell}{ }^{m}\right) e_{m} \\
& +\underbrace{\left.\sum_{\ell}\left(-\left[e_{k}, T_{i j}^{\ell} e_{\ell}\right]\right)+\pi^{*} d\left\langle T_{i j}^{\ell} e_{\ell}, e_{k}\right\rangle\right)}_{\pi^{*} d T_{i j k}-\sum_{\ell} \pi\left(e_{k}\right)\left(T_{i j}^{\ell}\right) e_{\ell}+\sum_{\ell, m} T_{i j}{ }^{\ell} T_{k \ell}{ }^{m} e_{m}} \\
= & \sum_{\ell} \pi\left(e_{\ell}\right)\left(T_{i j k}\right) \tilde{e}_{\ell}-\sum_{(i, j, k) \text { cyclic }} \sum_{\ell}\left(\pi\left(e_{i}\right)\left(T_{j k}^{\ell}\right) e_{\ell}-\sum_{m} T_{i j}{ }^{\ell} T_{k \ell}{ }^{m} e_{m}\right),
\end{aligned}
$$

where we have used that $\pi^{*} d f=\sum_{\ell} \pi\left(e_{\ell}\right)(f) \tilde{e}_{\ell}$ for all $f \in C^{\infty}(M)$. Therefore, for any $i, j, k, \ell$ fixed,

$$
\pi\left(e_{\ell}\right)\left(T_{i j k}\right) \tilde{e}_{\ell}-\sum_{(i, j, k) \text { cyclic }}\left(\pi\left(e_{i}\right)\left(T_{j k}{ }^{\ell}\right) e_{\ell}-\sum_{s} T_{i j}{ }^{s} T_{k s}{ }^{\ell} e_{\ell}\right)=0 .
$$

Taking now $i, j, k, \ell$ pairwise distinct, multiplying the above equality with $\gamma^{i j k}$ and summing over (pairwise distinct) $i, j, k, \ell$, we obtain

$$
4 \sum_{i, j, k, \ell}^{\prime} \pi\left(e_{\ell}\right)\left(T_{i j k}\right) \gamma^{\ell i j k}+3 \sum_{i, j, k, \ell, m}^{\prime} T_{i j}^{\ell} T_{k \ell m} \gamma^{m i j k}=0
$$

which is precisely (48) after re-organising the indices. We proved relation (47) which implies in particular that $\not^{2} \in C^{\infty}(U)$. From Lemma 42, $\not d$ is a Dirac generating operator for $E$.

Combining Proposition 40 with Theorem 41 we obtain:

Corollary 46. Let $E$ be a regular Courant algebroid on a manifold $M$ and $S$ a spinor bundle over $\mathrm{Cl}(E)$. For any sufficiently small open subset $U \subset M$, the set of Dirac generating operators for $\left.E\right|_{U}$ on $\left.S\right|_{U}$ has the structure of an affine space modelled on the vector space

$$
\left.V_{\not d}\right|_{U}:=\left\{e \in \Gamma\left(\left.E\right|_{U}\right),\left[\not d, \gamma_{e}\right] \in C^{\infty}(U)\right\}
$$

where $\not d$ is an arbitrarily chosen Dirac generating operator on $\left.S\right|_{U}$. 


\section{The canonical Dirac generating operator}

Let $E$ be a regular Courant algebroid with scalar product of neutral signature $(n, n)$. In this section we construct a canonical Dirac generating operator $d_{\mathbb{S}}$ on a suitable spinor bundle $\mathbb{S}$ of $E$, of the form (42). Its definition will involve an arbitrary generalized connection $D$ on $E$. By canonical we mean that $\phi_{\mathbb{S}}$ is independent of the choice of $D$.

We start with an arbitrary spinor bundle $S$ over $\mathrm{Cl}(E)$. Let $D$ a generalized connection on $E$ and $D^{S}$ a compatible $E$-connection on $S$. We begin by analyzing the dependence of $\not^{S}+\frac{1}{4} \gamma_{T^{D}}$ on the data $\left(D, D^{S}\right)$, where $\not^{S}$ is the Dirac operator defined by (41) Let $D^{\prime}=D+A$ be another generalized connection on $E$, where $A \in \Gamma\left(E^{*} \otimes \mathfrak{s o}(E)\right)$.

Proposition 47. The following holds.

(i) The torsions $T^{\prime}$ and $T$ of $D^{\prime}$ and $D$ are related by:

$$
T^{\prime}=T+\alpha
$$

where $\alpha \in \Gamma\left(\wedge^{3} E^{*}\right)$ is given by

$$
\alpha(u, v, w)=\sum_{(u, v, w) \text { cyclic }}\left\langle A_{u} v, w\right\rangle .
$$

(ii) The E-connection

$$
\left(D^{S}\right)^{\prime}:=D^{S}-\frac{1}{2} A
$$

is compatible with the generalized connection $D^{\prime}$. Here $A$ is considered as a map $E \rightarrow \wedge^{2} E^{*} \cong \wedge^{2} E \subset \mathrm{Cl}(E)$, so that $A_{e}$ acts by Clifford multiplication on $S$ for all $e \in E$.

(iii) The Dirac operators $\not D^{S}$ and $\left(\not D^{S}\right)^{\prime}$ associated with $\left(D, D^{S}\right)$ and $\left(D^{\prime}\right.$, $\left.\left(D^{S}\right)^{\prime}\right)$ are related by

$$
\left(\not D^{S}\right)^{\prime}=\not D^{S}-\frac{1}{4} \gamma_{\alpha}-\frac{1}{4} \gamma_{v_{A}}
$$

where $v_{A}=\operatorname{tr}_{\langle\cdot, \cdot\rangle} A=\sum A_{e_{i}} \tilde{e}_{i} \in \Gamma(E)$.

(iv) The operators $\not_{S}=\not D^{S}+\frac{1}{4} \gamma_{T}$ and $\not_{S}^{\prime}=\left(\not D^{S}\right)^{\prime}+\frac{1}{4} \gamma_{T^{\prime}}$ are related by

$$
\phi_{S}^{\prime}=\phi_{S}-\frac{1}{4} \gamma_{v_{A}} .
$$


Proof. (i) is relation (7).

(ii) To check the compatibility let $e, v \in \Gamma(E)$ and $s \in \Gamma(S)$ :

$$
\begin{aligned}
\left(D^{S}\right)_{e}^{\prime}(v s) & =D_{e}^{S}(v s)-\frac{1}{2} A_{e}(v s)=D_{e}(v) s+v D_{e}^{S} s-\frac{1}{2}\left(A_{e} v\right) s \\
& =D_{e}(v) s+v D_{e}^{S} s-\frac{1}{2}\left[A_{e}, v\right] s-\frac{1}{2} v A_{e} s \\
& =D_{e}^{\prime}(v) s+v\left(D^{S}\right)_{e}^{\prime} s .
\end{aligned}
$$

In the fourth equality we used that the commutator $\left[A_{e}, v\right] \in \Gamma(E)$ in the Clifford algebra is related to the evaluation $A_{e}(v)$ of $A_{e} \in \Gamma(\mathfrak{s o}(E))$ on $v$ by the formula

$$
A_{e}(v)=-\frac{1}{2}\left[A_{e}, v\right]
$$

(iii) With respect to an orthonormal frame $\left(e_{i}\right)$ we write

$$
A=\frac{1}{2} \sum_{i, j, k} A^{i j k} e_{i} \otimes\left(e_{j} \wedge e_{k}\right), \alpha=\frac{1}{6} \sum_{i, j, k} \alpha^{i j k} e_{i} \wedge e_{j} \wedge e_{k}
$$

where $A^{i j k}:=A\left(\tilde{e}_{i}, \tilde{e}_{j}, \tilde{e}_{k}\right)$ and $\alpha^{i j k}:=\alpha\left(\tilde{e}_{i}, \tilde{e}_{j}, \tilde{e}_{k}\right)$, where $E$ and $E^{*}$ are identified with the help of the scalar product. In particular, $A_{e_{i}}=\frac{1}{2} \epsilon_{i} \sum_{j, k} A^{i j k} e_{j} \wedge$ $e_{k}$ is identified with $\frac{1}{2} \epsilon_{i} \sum A^{i j k} e_{j} e_{k}$ in $\mathrm{Cl}(E)$. Similarly, $\alpha$ is identified with $\frac{1}{6} \sum \alpha^{i j k} e_{i} e_{j} e_{k}$ in $\mathrm{Cl}(E)$. With this notation,

$$
\begin{aligned}
\left(\not D^{S}\right)^{\prime}-\not D^{S} & =\frac{1}{2} \sum_{i} \tilde{e}_{i}\left(-\frac{1}{2} A_{e_{i}}\right)=-\frac{1}{4} \sum_{i} \tilde{e}_{i} A_{e_{i}}=-\frac{1}{8} \sum_{j \neq k} A^{i j k} \gamma_{i j k} \\
& =-\frac{1}{8} \sum_{i, j, k}^{\prime} A^{i j k} \gamma_{i j k}-\frac{1}{4} \sum_{j, k} A^{j j k} \epsilon_{j} \gamma_{k} .
\end{aligned}
$$

Remark that

$$
\sum_{i, j, k}^{\prime} A^{i j k} \gamma_{i j k}=\frac{1}{3} \sum_{i, j, k}^{\prime}\left(\sum_{(i, j, k) \text { cyclic }} A^{i j k} \gamma_{i j k}\right)=\frac{1}{3} \sum_{i, j, k}^{\prime} \alpha^{i j k} \gamma_{i j k}=2 \gamma_{\alpha}
$$

(where in the second equality we used (50) ) and similarly

$$
\sum_{j, k} A^{j j k} \epsilon_{j} \gamma_{k}=\sum_{j, k}\left\langle A\left(e_{j}, \tilde{e}_{j}\right), \tilde{e}_{k}\right\rangle \gamma_{k}=\gamma_{v_{A}}
$$

Combining the above relations we obtain (51). (A formula equivalent to (51) is stated as equation (2.22) in [10].)

(iv) follows from (49) and (51). 
The operator $\phi_{S}=\not D^{S}+\frac{1}{4} \gamma_{T^{D}}$, defined using a generalized connection $D$, depends on the choice of $E$-connection $D^{S}$ compatible with $D$. In order to remove this freedom we define

$$
\mathcal{S}:=S \otimes\left|\operatorname{det} S^{*}\right|^{\frac{1}{r_{S}}},
$$

where $r_{S}:=\operatorname{rank} S$ and $\left|\operatorname{det} S^{*}\right|^{\frac{1}{r_{S}}}$ denotes the bundle of $\frac{1}{r_{S}}$-densities of $S$. We call the bundle $\mathcal{S}$ the canonical spinor bundle of $S$.

Remark 48. i) For a vector bundle $V$ of rank $n$ and $s \in \mathbb{R}$, we denote by $\left|\operatorname{det} V^{*}\right|^{s}$ the line bundle of $s$-densities on $V$, whose fiber over $p \in M$ consists of all maps $\omega_{p}: \Lambda^{n} V_{p} \backslash\{0\} \rightarrow \mathbb{R}$ (called $s$-densities) which satisfy $\omega_{p}(\lambda \vec{v})=|\lambda|^{s} \omega_{p}(\vec{v})$, for any $\vec{v} \in \Lambda^{n} V_{p} \backslash\{0\}$ and $\lambda \in \mathbb{R} \backslash\{0\}$. Note that, when $s$ is an integer, $\left|\operatorname{det} V^{*}\right|^{s}$ is canonically isomorphic to $\left|\operatorname{det} V^{*}\right|^{\otimes s}$ and $\left|\operatorname{det} V^{*}\right|^{2 s}$ to $\left(\operatorname{det} V^{*}\right)^{2 s}$. Also,

$$
\left.\left|\operatorname{det} \Lambda V^{*}\right| \cong\left|\operatorname{det} V^{*}\right|^{N / 2}, \mid \operatorname{det}\left(V_{1} \otimes V_{2}\right)^{*}\right)\left.\right|^{s} \cong\left|\operatorname{det} V_{1}^{*}\right|^{s n_{2}} \otimes\left|\operatorname{det} V_{2}^{*}\right|^{s n_{1}} \text {, }
$$

where $N:=\operatorname{rank} \Lambda V, V_{i}$ are vector bundles of rank $n_{i}$ and $s \in \mathbb{R}$.

ii) An $E$-connection $D$ on $V$ induces an $E$-connection on $\operatorname{det} V^{*}$ and on $\left|\operatorname{det} V^{*}\right|^{s}$, for any $s \in \mathbb{R}$. The latter is defined as follows: if $D_{u} \omega=\alpha(u) \omega$, where $u \in \Gamma(E)$ and $\omega \in \Gamma\left(\operatorname{det} V^{*}\right)$, then $D_{u}\left(|\omega|^{s}\right)=s \alpha(u)|\omega|^{s}$, where $|\omega|^{s}$ is the $s$-density defined by $|\omega|^{s}\left(v_{1}, \cdots, v_{n}\right):=\left|\omega\left(v_{1}, \cdots, v_{n}\right)\right|^{s}$. Remark that if $D^{\prime}=D+A$ are two $E$-connections on $V$ then the induced $E$-connections on $\left|\operatorname{det} V^{*}\right|^{s}$ are related by $D_{u}^{\prime}=D_{u}-s \operatorname{trace} A_{u}$.

iii) Let $S$ and $S^{\prime}$ be two irreducible spinor bundles of $E$. Since $\langle\cdot, \cdot\rangle$ has neutral signature, we can write $S^{\prime}=S \otimes L$, where $L$ is a line bundle. From the second relation (55) we obtain that $\mathcal{S}^{\prime}=\mathcal{S} \otimes L \otimes\left|\operatorname{det} L^{*}\right|$. If $S$ and $S^{\prime}$ are isomorphic, then $L$ is trivializable, i.e. orientable. If $L$ is moreover oriented, then $L \otimes\left|\operatorname{det} L^{*}\right| \cong L \otimes L^{*}$ is canonically trivial. We deduce that $\mathcal{S}$ and $\mathcal{S}^{\prime}$ are then canonically isomorphic. This is another reason why $\mathcal{S}$ is called the canonical spinor bundle.

Lemma 49. Let $D$ be a generalized connection on $E$ with torsion $T$ and $D^{S}$ an E-connection on $S$ compatible with $D$. Then $D^{S}$ induces a connection $D^{\mathcal{S}}$ on $\mathcal{S}$, which is compatible with $D$ and depends only on $D$. In particular, $\phi_{\mathcal{S}}: \Gamma(\mathcal{S}) \rightarrow \Gamma(\mathcal{S})$ depends only on $D$.

Proof. It is clear that the $E$-connection $D^{\mathcal{S}}$ on $\mathcal{S}$ induced by $D^{S}$ is again compatible with $D$. Any other $E$-connection on $S$ compatible with $D$ is of the form $\left(D^{S}\right)^{\prime}=D^{S}+\varphi \otimes \operatorname{Id}_{S}$ for some section $\varphi \in \Gamma\left(E^{*}\right)$. From Remark 48 ii) $D^{S}$ and $\left(D^{S}\right)^{\prime}$ induce the same connection on $\mathcal{S}$. 
In order to define a Dirac generating operator independent of $D$ we consider as in [10] the following canonical weighted spinor bundle

$$
\mathbb{S}:=\mathcal{S} \otimes L, \quad L:=\left|\operatorname{det} T^{*} M\right|^{\frac{1}{2}} .
$$

The line bundle $L$ carries an induced $E$-connection $D^{L}$ defined by

$$
D_{v}^{L} \mu:=\mathcal{L}_{\pi(v)} \mu-\frac{1}{2} \operatorname{div}_{D}(v) \mu,
$$

where $v \in \Gamma(E), \mathcal{L}_{\pi(v)} \mu$ denotes the Lie derivative of the densitity $\mu \in \Gamma(L)$ with respect to the vector field $\pi(v)$ and $\operatorname{div}_{D}(v):=\operatorname{tr} D v$.

Remark 50. The Lie derivative $\mathcal{L}_{X} \mu$ of a density $\mu \in \Gamma\left(\left|\operatorname{det} T^{*} M\right|^{1 / r}\right)$ in the direction of $X \in \mathfrak{X}(M)$ is defined by $\mathcal{L}_{X} \mu=\frac{1}{2 r} \alpha(X) \mu$, when $\mathcal{L}_{X} \mu^{2 r}=$ $\alpha(X) \mu^{2 r}$. In the latter expression $\mathcal{L}_{X}$ denotes the Lie derivative on $\left(\operatorname{det} T^{*} M\right)^{2}$ (defined by $\mathcal{L}_{X}\left(\omega^{2}\right)=2\left(\mathcal{L}_{X} \omega\right) \omega$ where $\mathcal{L}_{X} \omega$ is the Lie derivative of the volume form $\omega$ of $M$ ). If $\nabla$ is a torsion-free connection on $M$, then it induces a connection (also denoted by $\nabla$ ) on $\left|\operatorname{det} T^{*} M\right|^{1 / r}$ and one can show that

$$
\nabla_{X} \mu=\mathcal{L}_{X} \mu-\frac{1}{r} \operatorname{trace}(\nabla X) \mu, \forall \mu \in \Gamma\left(\left|\operatorname{det} T^{*} M\right|^{1 / r}\right), \quad X \in \mathfrak{X}(M) .
$$

Lemma 51. If $D$ is the generalized connection from Lemma 45, then the E-connection $D^{L}$ defined by (57) is induced by a usual connection on $L$.

Proof. We need to show that $\operatorname{div}_{D}(v)=0$ for any $v \in \Gamma(\operatorname{ker} \pi)$ (see Example 5). Consider the frame $\left(p_{a}, q_{a}\right)$ constructed after Lemma 43 and recall that it is parallel with respect to the connection $\nabla_{\pi(e)}=D_{e}$ on $E$. Its dual frame is $\left(\tilde{p}_{a}, \tilde{q}_{b}\right)=\left(q_{a}, p_{b}\right)$ and

$\operatorname{div}_{D}(v)=\sum_{a}\left(\left\langle D_{p_{a}}(v), q_{a}\right\rangle+\left\langle D_{q_{a}}(v), p_{a}\right\rangle\right)=\sum_{a}\left\langle\nabla_{\pi\left(q_{a}\right)}(v), p_{a}\right\rangle=\sum_{a} \pi\left(q_{a}\right)\left\langle v, p_{a}\right\rangle$

where we used $\pi\left(p_{a}\right)=0$ and $\nabla p_{a}=0$. From Corollary 44 we obtain $\operatorname{div}_{D}(v)=0$ as needed.

Theorem 52. [1] Let $D$ be a generalized connection on $E$ with torsion $T$, $D^{\mathbb{S}}=D^{\mathcal{S}} \otimes D^{L}$ the induced compatible E-connection on the canonical weighted spinor bundle $\mathbb{S}$, and $\not D^{\mathbb{S}}$ the corresponding Dirac operator on $\mathbb{S}$. Then

$$
\not_{\mathbb{S}}=\not D^{\mathbb{S}}+\frac{1}{4} \gamma_{T}: \Gamma(\mathbb{S}) \rightarrow \Gamma(\mathbb{S})
$$

is a Dirac generating operator, independent of $D$. 
Proof. Replacing $D$ by another generalized connection $D^{\prime}=D+A$ changes $D^{S}$ to $\left(D^{\prime}\right)^{S}=D^{S}-\frac{1}{2} A$ (see Proposition 47). This implies that $\left(D^{\prime}\right)^{\mathcal{S}}=$ $D^{\mathcal{S}}-\frac{1}{2} A$. Here we use that the Clifford action of $A_{u}$ on $S$ is trace-free (see the next remark) and Remark 48 ii). From Proposition 47 again (applied to the spinor bundle $\mathcal{S}$ ) we obtain that $\not D^{\mathcal{S}}+\frac{1}{4} \gamma_{T}$ changes to $\left(\not D^{\mathcal{S}}\right)^{\prime}+\frac{1}{4} \gamma_{T^{\prime}}=$ $\left(\not D^{\mathcal{S}}+\frac{1}{4} \gamma_{T}\right)-\frac{1}{4} \gamma_{v_{A}}$. (See Proposition 47 for the definition of $v_{A}$.)

On the other hand, on $\mathbb{S}=\mathcal{S} \otimes L$,

$$
\not D(s \otimes l)=\left(\not D^{\mathcal{S}} s\right) \otimes l+\frac{1}{2} \tilde{e}_{i} s \otimes D_{e_{i}}^{L} l, s \in \Gamma(\mathcal{S}), l \in \Gamma(L)
$$

and a similar expression holds for the Dirac operator $\not D^{\prime}$ on $\mathbb{S}$ computed with the generalized connection $D^{\prime}$. We deduce that

$$
\begin{aligned}
& \left(\not D+\frac{1}{4} \gamma_{T}\right)(s \otimes l)=\left(\not D^{\mathcal{S}} s+\frac{1}{4} T s\right) \otimes l+\frac{1}{2} \tilde{e}_{i} s \otimes D_{e_{i}}^{L} l \\
& \left(\not D^{\prime}+\frac{1}{4} \gamma_{T^{\prime}}\right)(s \otimes l)=\left(\not D^{\mathcal{S}} s+\frac{1}{4} T s-\frac{1}{4} v_{A} s\right) \otimes l+\frac{1}{2} \tilde{e}_{i} s \otimes\left(D^{\prime}\right)_{e_{i}}^{L} l
\end{aligned}
$$

But $\operatorname{div}_{D^{\prime}}=\operatorname{div}_{D}-\left\langle v_{A}, \cdot\right\rangle$ implies that $\left(D^{\prime}\right)_{v}^{L} l=D_{v}^{L} l+\frac{1}{2}\left\langle v_{A}, v\right\rangle l$. We deduce that (59) is independent on $D$.

It remains to show that $\phi_{\mathbb{S}}$ is a Dirac generating operator. As this is a local property, we need to show that for any sufficiently small open subset $U \subset M$, the restriction $\left.\phi_{\mathbb{S}}\right|_{U}: \Gamma\left(\left.\mathbb{S}\right|_{U}\right) \rightarrow \Gamma\left(\left.\mathbb{S}\right|_{U}\right)$ is a Dirac generating operator. Choose $U$ like in Lemma 45, and let $D$ be the generalized connection on $\left.E\right|_{U}$ defined by the flat metric connection $\nabla$ used in that lemma. From Lemma 51, $D^{\mathcal{S}} \otimes D^{L}$ on $\left.\mathbb{S}\right|_{U}=\left.(\mathcal{S} \otimes L)\right|_{U}$ is induced by a usual connection compatible with $\nabla$. Since $\phi_{\mathbb{S}}$ is independent on $D$, we deduce that on $U$ it coincides with the operator constructed in Lemma 45. In particular, it is a Dirac generating operator.

Remark 53. In the proof we have used the fact that any representation of $\mathfrak{s p i n}(k, \ell) \subset \mathrm{Cl}\left(\mathbb{R}^{k, \ell}\right)$ induced by a representation of the Clifford algebra $\mathrm{Cl}\left(\mathbb{R}^{k, \ell}\right)$ is trace-free. This follows from the fact that the Lie algebra $\mathfrak{s p i n}(k, \ell)$ is spanned by commutators $u v-v u$, where $u, v \in \mathbb{R}^{k, \ell}$.

Definition 54. The operator $\phi_{\mathbb{S}}: \Gamma(\mathbb{S}) \rightarrow \Gamma(\mathbb{S})$ is called the canonical Dirac generating operator associated to $E$.

Remark 55. Our canonical Dirac generating operator coincides with the Dirac generating operator constructed in Theorem 4.1 of [1]. This follows from formula (53) of [1], by noticing a difference of a minus sign between our definition for the torsion of a generalized connection and that from [1] (see 
Section 3.2 of this reference). Our Dirac generating operator also coincides (up to a multiplicative constant factor) with the Dirac generating operator from Proposition 5.12 of [15] (in formula (5.14) of [15] the term $\frac{1}{2} \gamma\left(C_{\nabla}\right)$ should have a minus sign).

\section{Standard form of the canonical Dirac gen- erating operator}

In this section we provide an expression for the canonical Dirac generating operator $\phi_{\mathbb{S}}$ which uses the structure of regular Courant algebroids (see [7]). We consider the setting from the previous section. For simplicity, from now on $\phi_{\mathbb{S}}$ will be denoted by $\not$. From Lemma 43 , there is a vector bundle isomorphism

$$
I: E \rightarrow F^{*} \oplus \mathcal{G} \oplus F,
$$

where we recall that $F=\pi(E) \subset T M$ is an integrable distribution and $\mathcal{G} \subset$ ker $\pi \subset E$ is a subbundle. The isomorphism $I$ maps the anchor $\pi: E \rightarrow T M$ of $E$ to the map $\rho(\xi+r+X)=X$ and the scalar product of $E$ to a scalar product

$$
\langle\xi+r+X, \eta+s+y\rangle=\frac{1}{2}(\xi(Y)+\eta(X))+(r, s)^{\mathcal{G}},
$$

where $\xi+r+X, \eta+s+Y \in F^{*} \oplus \mathcal{G} \oplus F$, and the scalar product $(\cdot, \cdot)^{\mathcal{G}}$ on $\mathcal{G}$ is of neutral signature. The bundle $\mathcal{G}$, together with $(\cdot, \cdot)^{\mathcal{G}}$, is canonically associated to $E$. More precisely, $\left(\mathcal{G},(\cdot, \cdot)^{\mathcal{G}}\right)$ is isomorphic to $(\operatorname{ker} \pi) /(\operatorname{ker} \pi)^{\perp}$ with scalar product induced by the scalar product of $E$. Moreover, $\mathcal{G}$ is a bundle of Lie algebras, with Lie bracket $[\cdot, \cdot]^{\mathcal{G}}$ induced from the Dorfman bracket of $E$. The scalar product $(\cdot, \cdot)^{\mathcal{G}}$ is invariant with respect to $[\cdot, \cdot]^{\mathcal{G}}$, that is the adjoint representation of the Lie algebra is by skew-symmetric endomorphisms. In fact, these properties follow immediately from $\mathcal{G} \subset \operatorname{ker} \pi$. An isomorphism (60) as above is called a dissection of $E$ [7].

The Dorfman bracket $[\cdot, \cdot]$ of $F^{*} \oplus \mathcal{G} \oplus F$ induced from $E$ via a dissection satisfies

$$
\operatorname{Pr}_{\mathcal{G}}\left[r_{1}, r_{2}\right]=\left[r_{1}, r_{2}\right]^{\mathcal{G}}, r_{i} \in \Gamma(\mathcal{G}),
$$

where $\operatorname{Pr}_{\mathcal{G}}$ is the natural projection from $F^{*} \oplus \mathcal{G} \oplus F$ on $\mathcal{G}$ (we shall use a similar notation $\operatorname{Pr}_{F^{*}}$ for the natural projection on $F^{*}$ ).

Therefore, we may (and will) assume that the given regular Courant algebroid is of the form $E=F^{*} \oplus \mathcal{G} \oplus F$, with anchor $\rho(\xi+r+X)=X$, metric given by (61) and Dorfman bracket $[\cdot, \cdot]$ satisfying (62). As proved in 
Lemma 2.1 of [7], the Dorfman bracket of $E$ is determined by its components

$$
\begin{aligned}
& \nabla: \Gamma(F) \times \Gamma(\mathcal{G}) \rightarrow \Gamma(\mathcal{G}), \nabla_{X}(r):=\operatorname{Pr}_{\mathcal{G}}[X, r] \\
& R: \Gamma(F) \times \Gamma(F) \rightarrow \Gamma(\mathcal{G}), R(X, Y):=\operatorname{Pr}_{\mathcal{G}}[X, Y] \\
& \mathcal{H}: \Gamma(F) \times \Gamma(F) \times \Gamma(F) \rightarrow C^{\infty}(M), \mathcal{H}(X, Y, Z):=\left(\operatorname{Pr}_{F^{*}}[X, Y]\right)(Z) .
\end{aligned}
$$

Note that here $[X, Y]$ stands for the Dorfman bracket

$$
[X, Y]=\mathcal{H}(X, Y, \cdot)+R(X, Y)+\mathcal{L}_{X} Y
$$

of $X, Y$ as sections of $F \subset E$, whereas, for the rest of this section, the Lie bracket of vector fields will be always denoted by $\mathcal{L}_{X} Y$. The map $\nabla$ is a partial connection on $\mathcal{G}$, the map $R$ is a 2 -form on $F$ with values in $\mathcal{G}$ and $\mathcal{H}$ is a 3 -form on $F$. The properties of the triple $(\nabla, R, \mathcal{H})$ are described in Theorem 2.3 of [7].

The next lemma was proved in [7] and can be checked directly (we remark a difference of sign between our definition for the torsion of a generalized connection and that from [7]). By a torsion-free connection on $F$ we mean a partial connection $\nabla^{F}: \Gamma(F) \times \Gamma(F) \rightarrow \Gamma(F)$ which satisfies $\nabla_{X} Y-\nabla_{Y} X=$ $\mathcal{L}_{X} Y$ for any $X, Y \in \Gamma(F)$.

Lemma 56. ([7]) Let $\nabla^{F}$ be a torsion-free connection on $F$. Then

$$
\nabla_{\xi+r+X}^{E}(\eta+s+Y):=\left(\nabla_{X}^{F} \eta-\frac{1}{3} \mathcal{H}(X, Y, \cdot), \nabla_{X} s+\frac{2}{3}[r, s]^{\mathcal{G}}, \nabla_{X}^{F} Y\right)
$$

is a generalized connection on $E$ with torsion given by

$$
\begin{aligned}
T^{\nabla^{E}}(u, v, w)= & -\mathcal{H}(X, Y, Z)-(R(X, Y), t)^{\mathcal{G}}-(R(Y, Z), r)^{\mathcal{G}}-(R(Z, X), s)^{\mathcal{G}} \\
& +\left([r, s]^{\mathcal{G}}, t\right)^{\mathcal{G}},
\end{aligned}
$$

for any $u=\xi+r+X, v=\eta+s+Y, w=\zeta+t+Z$ (where $\xi, \eta, \zeta \in F^{*}$, $r, s, t \in \mathcal{G}, X, Y, Z \in F)$.

Remark 57. For any regular Courant algebroid $E$ with anchor $\pi: E \rightarrow$ $T M$, the quotient $\mathcal{A}:=E /(\operatorname{ker} \pi)^{\perp}$ inherits a Lie algebroid structure from the Dorfman bracket of $E$. This Lie algebroid is called in [7] the ample Lie algebroid associated to $E$. A dissection induces a bundle isomorphism $\mathcal{A} \cong \mathcal{G} \oplus F$ and a Lie algebroid structure on $\mathcal{G} \oplus F$ (inherited from the Lie algebroid structure of $\mathcal{A}$ ). The restriction of the 3 -form $\Omega:=-T^{\nabla^{E}}$ to $\mathcal{G} \oplus F$ is closed with respect to the Lie algebroid differential of $\mathcal{G} \oplus F$ and its cohomology class is independent on the chosen dissection. It is called the Severa class of $E$, as it coincides with the Severa class when $E$ is exact [7]. 
Let $\Lambda F^{*}$ be the bundle of forms over $F$. It is a spinor bundle over the bundle of Clifford algebras $\mathrm{Cl}\left(F^{*} \oplus F\right)$, where $F^{*} \oplus F$ has scalar product $\langle\xi+X, \eta+Y\rangle=\frac{1}{2}(\eta(X)+\xi(Y))$. The Clifford representation is

$$
(X+\xi) \cdot \alpha:=\iota_{X} \alpha+\xi \wedge \alpha
$$

where $\iota_{X} \alpha:=\alpha(X, \cdot)$ denotes the interior product. Let $S_{\mathcal{G}}$ be an irreducible spinor bundle over $\mathrm{Cl}(\mathcal{G})$, where $\mathcal{G}$ is considered with the scalar product $(\cdot, \cdot)^{\mathcal{G}}$. We assume that $\mathcal{G}$ is oriented, so that $S_{\mathcal{G}}$ is $\mathbb{Z}_{2^{-}}$graded. The $\mathbb{Z}_{2^{-}}$ graded tensor product $S:=\Lambda F^{*} \hat{\otimes} S_{\mathcal{G}}$ is an irreducible spinor bundle over $\mathrm{Cl}(E)=\mathrm{Cl}\left(F^{*} \oplus F\right) \hat{\otimes} \mathrm{Cl}(\mathcal{G})$. (Basic facts concerning the $\mathbb{Z}_{2}$-graded tensor product are reviewed in more detail in Appendix 11, in particular, see relation (88) for the Clifford action of $E$ on $S$.)

Lemma 58. The canonical weighted spinor bundle of $S$ is given by

$$
\mathbb{S}=\left(\Lambda F^{*} \otimes|\operatorname{det}(\operatorname{Ann} F)|^{1 / 2}\right) \hat{\otimes} \mathcal{S}_{\mathcal{G}}
$$

where Ann $F \subset T^{*} M$ is the annihilator of $F$ and $\mathcal{S}_{\mathcal{G}}=S_{\mathcal{G}} \otimes\left|\operatorname{det} S_{\mathcal{G}}^{*}\right|^{1 / g}$ is the canonical spinor bundle of $S_{\mathcal{G}}$ (with $g:=\operatorname{rank} S_{\mathcal{G}}$ ).

Proof. The isomorphism (given by contraction) between $(\operatorname{det} T M) \otimes \operatorname{det} F^{*}$ and det $\left((\operatorname{Ann} F)^{*}\right)$, induces a canonical isomorphism

$$
|\operatorname{det} F|^{1 / 2} \otimes\left|\operatorname{det} T^{*} M\right|^{1 / 2} \cong|\operatorname{det}(\operatorname{Ann} F)|^{1 / 2} \text {. }
$$

The claim follows from the definition of the canonical weighted spinor bundle $\mathbb{S}$, recall (56) and (54), combined with relations (55) and (67).

Recall the definition of the $E$-connection $D^{\mathbb{S}}$ from Theorem 52 computed from a generalized connection $D$.

Lemma 59. The E-connection $\nabla^{\mathbb{S}}$ on $\mathbb{S}$ computed from the generalized connection $\nabla^{E}$ defined in Lemma 56 has the following expression: for any $\omega \in \Gamma\left(\Lambda F^{*}\right), \tau \in \Gamma\left(|\operatorname{det}(\operatorname{Ann} F)|^{1 / 2}\right)$ and $s \in \Gamma\left(\mathcal{S}_{\mathcal{G}}\right)$ we have

$$
\begin{aligned}
& \nabla_{u}^{\mathbb{S}}(\omega \otimes \tau \otimes s)=\left(\nabla_{X}^{F} \omega\right) \otimes \tau \otimes s+\omega \otimes\left(\mathcal{L}_{X} \tau\right) \otimes s \\
& +\frac{1}{3}\left(\iota_{X} \mathcal{H}\right) \wedge \omega \otimes \tau \otimes s+\omega \otimes \tau \otimes\left(\nabla_{X}^{0, \mathcal{S}_{\mathcal{G}}} s-\frac{1}{3}\left(\operatorname{ad}_{r}\right)(s)\right) .
\end{aligned}
$$

Above $u=\xi+r+X, \mathcal{L}_{X} \tau$ denotes the Lie derivative of $\tau$ in the direction of $X \in \Gamma(F), \nabla^{0, \mathcal{S}_{\mathcal{G}}}$ is an $F$-connection on $\mathcal{S}_{\mathcal{G}}$, induced by an $F$-connection $\nabla^{0, S_{\mathcal{G}}}$ on $S_{\mathcal{G}}$ compatible with the $F$-connection $\nabla$ of $\mathcal{G}$ and $\operatorname{ad}_{r} \in \mathfrak{s o}(\mathcal{G})$ is considered as a 2-form on $\mathcal{G}$, which acts by Clifford multiplication on $\mathcal{S}_{\mathcal{G}}$. 
Proof. We remark that $\nabla^{E}=\nabla^{F^{*}+F}+\nabla^{\mathcal{G}}$, where $\nabla^{F^{*}+F}$ and $\nabla^{\mathcal{G}}$ are $E$ connections on $F^{*} \oplus F$ and $\mathcal{G}$ respectively, defined by

$$
\begin{aligned}
& \nabla_{u}^{F^{*}+F}(\eta+Y)=\nabla_{X}^{F} \eta-\frac{1}{3} \mathcal{H}(X, Y, \cdot)+\nabla_{X}^{F} Y, \\
& \nabla_{u}^{\mathcal{G}}(s):=\nabla_{X} s+\frac{2}{3} \operatorname{ad}_{r}(s)
\end{aligned}
$$

where $\operatorname{ad}_{r}(s)=[r, s]^{\mathcal{G}}$. The $F$-connection $\nabla^{F}$ induces an $F$-connection (also denoted by $\nabla^{F}$ ) on $\Lambda F^{*}$. A straightforward computation shows that the E-connection

$$
\nabla_{u}^{F, \text { spin }}:=\nabla_{X}^{F}+\frac{1}{3}\left(\iota_{X} \mathcal{H}\right) \wedge
$$

on $\Lambda F^{*}$ is compatible with $\nabla^{F^{*}+F}$. On the other hand, like in the proof of Proposition 47 ii), the E-connection

$$
\nabla_{u}^{\mathcal{G}, \text { spin }}:=\nabla_{X}^{0, S_{\mathcal{G}}}-\frac{1}{3} \operatorname{ad}_{r}
$$

on $S_{\mathcal{G}}$ is compatible with $\nabla^{\mathcal{G}}$. We obtain that $\nabla^{F \text {,spin }} \otimes \nabla^{\mathcal{G} \text {, spin }}$ is an $E$ connection on $S=\Lambda F^{*} \hat{\otimes} S_{\mathcal{G}}$ compatible with $\nabla^{E}$. The definition of $\nabla^{E}$ implies that $\operatorname{div}_{\nabla^{E}}(u)=\operatorname{trace}\left(\nabla^{F} X\right)$ and thus

$$
\left(\nabla^{E}\right)_{u}^{L} \mu=\mathcal{L}_{X} \mu-\frac{1}{2} \operatorname{trace}\left(\nabla^{F} X\right) \mu
$$

for any section $\mu$ of $L=\left|\operatorname{det} T^{*} M\right|^{1 / 2}$. We obtain that the $E$-connection $\nabla^{\mathbb{S}}$ on $\mathbb{S}=\left(\Lambda F^{*} \otimes|\operatorname{det} F|^{1 / 2}\right) \hat{\otimes} \mathcal{S}_{\mathcal{G}} \otimes L$ is given by

$$
\nabla^{\mathbb{S}}:=\nabla^{F, \text { spin }} \otimes \nabla^{\mathcal{G}, \text { spin }} \otimes\left(\nabla^{E}\right)^{L}
$$

where we preserve the same symbols $\nabla^{F \text {, spin }}$ and $\nabla^{\mathcal{G} \text {, spin }}$ for the $E$-connections induced by $\nabla^{F \text {,spin }}$ and $\nabla^{\mathcal{G} \text {, spin }}$ on $\Lambda F^{*} \otimes|\operatorname{det} F|^{1 / 2}$ and $\mathcal{S}_{\mathcal{G}}$ respectively. In order to compute $\nabla^{\mathbb{S}}$ we shall compute $\nabla^{F, \operatorname{spin}} \otimes\left(\nabla^{E}\right)^{L}$ and $\nabla^{\mathcal{G} \text {, spin }}$ separately.

We begin with $\nabla^{F, \text { spin }} \otimes\left(\nabla^{E}\right)^{L}$. This is an E-connection on $\Lambda F^{*} \otimes$ $|\operatorname{det} F|^{1 / 2} \otimes L$. Relation (69) holds also on $\Lambda F^{*} \otimes|\operatorname{det} F|^{1 / 2}$, as the endomorphism $\omega \rightarrow \frac{1}{3}\left(\iota_{X} \mathcal{H}\right) \wedge \omega$ of $\Lambda F^{*}$ is trace-free. Let $\omega \in \Gamma\left(\Lambda F^{*}\right), l \in \Gamma\left(|\operatorname{det} F|^{1 / 2}\right)$ and $\mu \in \Gamma(L)$. Then

$$
\begin{aligned}
& \left(\nabla^{F, \text { spin }} \otimes\left(\nabla^{E}\right)^{L}\right)_{u}(\omega \otimes l \otimes \mu)=\nabla_{X}^{F, \text { spin }}(\omega \otimes l) \otimes \mu+\omega \otimes l \otimes\left(\nabla^{E}\right)_{X}^{L} \mu \\
& =\left(\nabla_{X}^{F}(\omega \otimes l)+\frac{1}{3}\left(i_{X} \mathcal{H} \wedge \omega\right) \otimes l\right) \otimes \mu+\omega \otimes l \otimes\left(\mathcal{L}_{X} \mu-\frac{1}{2} \operatorname{trace}\left(\nabla^{F} X\right) \mu\right) \\
& =\left(\nabla_{X}^{F} \omega\right) \otimes l \otimes \mu+\omega \otimes \mathcal{L}_{X}(l \otimes \mu)+\frac{1}{3}\left(\iota_{X} \mathcal{H} \wedge \omega\right) \otimes l \otimes \mu
\end{aligned}
$$


where in the last equality we used

$$
\begin{aligned}
& \nabla_{X}^{F}(\omega \otimes l)=\left(\nabla_{X}^{F} \omega\right) \otimes l+\omega \otimes \nabla_{X}^{F} l \\
& \nabla_{X}^{F} l=\mathcal{L}_{X} l+\frac{1}{2} \operatorname{trace}\left(\nabla^{F} X\right) l .
\end{aligned}
$$

(see (58) for the second relation (73) $)$.

Next, we compute $\nabla^{\mathcal{G}}$, spin . Relation (70) holds also on $\mathcal{S}_{\mathcal{G}}$ (with $\nabla^{0, S_{\mathcal{G}}}$ replaced by $\nabla^{0, \mathcal{S}_{\mathcal{G}}}$, the $E$-connection on $\mathcal{S}_{\mathcal{G}}$ induced by $\left.\nabla^{0, S_{\mathcal{G}}}\right)$. This follows from the fact that the Clifford action by $\operatorname{ad}_{r} \in \Gamma\left(\Lambda^{2} \mathcal{G}\right)$ on $S_{\mathcal{G}}$ is trace-free. The latter is a consequence of Remark 53. So we have proven:

$$
\nabla_{u}^{\mathcal{G}, \text { spin }}=\nabla_{X}^{0, \mathcal{S}_{\mathcal{G}}}-\frac{1}{3} \operatorname{ad}_{r} \quad \text { on } \quad \mathcal{S}_{\mathcal{G}} .
$$

Combining (71), (72) and (74), we obtain (68).

Notation 60. For any $\tau \in \Gamma\left(|\operatorname{det}(\operatorname{Ann} F)|^{1 / 2}\right), \mathcal{L}_{X} \tau$ is $C^{\infty}(M)$-linear in $X$, when $X \in \Gamma(F)$ (as $\mathcal{L}_{f X} \omega=f \mathcal{L}_{X} \omega$, for any $\omega \in \Gamma($ Ann $F)$ ). The map $\Gamma(F) \ni X \rightarrow \mathcal{L}_{X} \tau$ is a 1 -form on $F$ with values in $|\operatorname{det}(\operatorname{Ann} F)|^{1 / 2}$, which was denoted by $\mathcal{L}(\tau)$.

We arrive now at what we call the standard form for the canonical Dirac generating operator in terms of the data encoding the regular Courant algebroid.

Theorem 61. Let $E$ be a regular Courant algebroid with anchor $\pi: E \rightarrow T M$ and scalar product of neutral signature. In terms of a dissection $F^{*} \oplus \mathcal{G} \oplus F$ of $E$, the canonical Dirac generating operator is given by

$$
\begin{aligned}
\not d(\omega \otimes \tau \otimes s) & =\left(d^{F} \omega\right) \otimes \tau \otimes s+\mathcal{L}(\tau) \wedge \omega \otimes s+\nabla^{0, \mathcal{S}_{\mathcal{G}}}(s) \wedge \omega \otimes \tau \\
& -(\mathcal{H} \wedge \omega) \otimes \tau \otimes s+\frac{1}{4}(-1)^{|\omega|+1} \omega \otimes \tau \otimes C s \\
& +(-1)^{|\omega|+1} \bar{R}(\omega \otimes \tau \otimes s),
\end{aligned}
$$

where $\omega \in \Gamma\left(\Lambda F^{*}\right), \tau \in \Gamma\left(|\operatorname{det}(\operatorname{Ann} F)|^{1 / 2}\right)$ and $s \in \Gamma\left(\mathcal{S}_{\mathcal{G}}\right)$. Above $d^{F}$ : $\Gamma\left(\Lambda^{\bullet} F^{*}\right) \rightarrow \Gamma\left(\Lambda^{\bullet+1} F^{*}\right)$ is the exterior derivative along the integrable distribution $F=\operatorname{im} \pi, C \in \Gamma\left(\Lambda^{3} \mathcal{G}^{*}\right)$ is the Cartan form $C(u, v, w)=\left([u, v]^{\mathcal{G}}, w\right)^{\mathcal{G}}$ of $\mathcal{G}$ viewed as a section of $\mathrm{Cl}(\mathcal{G}), C$ s denotes its Clifford action on $s$ and

$$
\bar{R}(\omega \otimes \tau \otimes s)=\frac{1}{2} \sum_{i, j, k}\left(R\left(X_{i}, X_{j}\right), r_{k}\right)^{\mathcal{G}}\left(\alpha_{i} \wedge \alpha_{j} \wedge \omega\right) \otimes \tau \otimes \tilde{r}_{k} s
$$

where $\left(X_{i}\right)$ is a basis of $F,\left(\alpha_{i}\right)$ is the dual basis, i.e. $\alpha_{i}\left(X_{j}\right)=\delta_{i j},\left(r_{i}\right)$ is a basis of $\mathcal{G}$ and $\left(\tilde{r}_{i}\right)$ is the dual basis with respect to $(\cdot, \cdot)^{\mathcal{G}}$. 
Proof. The bases of $E$

$$
\left(e_{i}\right):=\left(X_{i}, r_{j}, \alpha_{k}\right), \quad\left(\tilde{e}_{i}\right):=\left(2 \alpha_{i}, \tilde{r}_{j}, 2 X_{k}\right)
$$

are dual with respect to the scalar product (61) of $E$. Using Lemma 59 and the definition of the Clifford action, we obtain

$$
\begin{aligned}
\sum_{i} \tilde{e}_{i} \nabla_{e_{i}}^{\mathbb{S}}(\omega \otimes \tau \otimes s) & =\sum_{i}\left(2\left(\alpha_{i} \wedge \nabla_{X_{i}}^{F} \omega\right) \otimes \tau \otimes s+2\left(\alpha_{i} \wedge \omega\right) \otimes \mathcal{L}_{X_{i}} \tau \otimes s\right. \\
& +\frac{2}{3}\left(\alpha_{i} \wedge \iota_{X_{i}} \mathcal{H} \wedge \omega\right) \otimes \tau \otimes s+2\left(\alpha_{i} \wedge \omega\right) \otimes \tau \otimes \nabla_{X_{i}}^{0, \mathcal{S}_{\mathcal{G}}} s \\
& \left.+(-1)^{|\omega|+1} \frac{1}{3} \omega \otimes \tau \otimes\left(\tilde{r}_{i} \operatorname{ad}_{r_{i}}\right)(s)\right) .
\end{aligned}
$$

But

$$
\begin{aligned}
& \sum_{i} \alpha_{i} \wedge \nabla_{X_{i}}^{F} \omega=d^{F} \omega, \sum_{i} \alpha_{i} \wedge \iota_{X_{i}} \mathcal{H}=3 \mathcal{H}, \sum_{i} \alpha_{i} \otimes \mathcal{L}_{X_{i}} \tau=\mathcal{L}(\tau), \\
& \sum_{i} \tilde{r}_{i} \operatorname{ad}_{r_{i}}=\frac{1}{2} \sum_{i, j, k}\left(\operatorname{ad}_{r_{i}}\left(r_{j}\right), r_{k}\right)^{\mathcal{G}} \tilde{r}_{i} \tilde{r}_{j} \tilde{r}_{k}=3 C
\end{aligned}
$$

where in the first equality (78) we used that $\nabla^{F}$ is torsion-free and the last equality holds in the Clifford algebra $\mathrm{Cl}(\mathcal{G})$. We obtain that

$$
\begin{aligned}
& \frac{1}{2} \sum_{i} \tilde{e}_{i} \nabla_{e_{i}}^{\mathbb{S}}(\omega \otimes \tau \otimes s)=\left(d^{F} \omega\right) \otimes \tau \otimes s+\mathcal{L}(\tau) \wedge \omega \otimes s \\
& +\left(\nabla^{0, \mathcal{S}_{\mathcal{G}}} s\right) \wedge \omega \otimes \tau+(\mathcal{H} \wedge \omega) \otimes \tau \otimes s+\frac{1}{2}(-1)^{|\omega|+1} \omega \otimes \tau \otimes C s .
\end{aligned}
$$

From (179) and the definition of $\not d$ we obtain

$$
\begin{aligned}
& \not(\omega \otimes \tau \otimes s)=\left(d^{F} \omega\right) \otimes \tau \otimes s+\mathcal{L}(\tau) \wedge \omega \otimes s+\left(\nabla^{0, \mathcal{S}_{\mathcal{G}}} s\right) \wedge \omega \otimes \tau \\
& +(\mathcal{H} \wedge \omega) \otimes \tau \otimes s+\frac{1}{2}(-1)^{|\omega|+1} \omega \otimes \tau \otimes C s+\frac{1}{4} T^{\nabla^{E}}(\omega \otimes \tau \otimes s) .
\end{aligned}
$$

In order to conclude our proof we need to express $T^{\nabla^{E}}$ as a section of $\Lambda^{3} E$ and to compute $T^{\nabla^{E}}(\omega \otimes \tau \otimes s)$, where $T^{\nabla^{E}} \in \Gamma\left(\Lambda^{3} E\right) \subset \Gamma(\mathrm{Cl}(E))$ acts by Clifford multiplication on $\omega \otimes \tau \otimes s$. Using the bases (77), we write

$$
\begin{aligned}
T^{\nabla^{E}}= & \frac{1}{6} \sum_{i, j, k} T^{\nabla^{E}}\left(e_{i}, e_{j}, e_{k}\right) \tilde{e}_{i} \wedge \tilde{e}_{j} \wedge \tilde{e}_{k} \\
= & \frac{4}{3} \sum_{i, j, k} T^{\nabla^{E}}\left(X_{i}, X_{j}, X_{k}\right) \alpha_{i} \wedge \alpha_{j} \wedge \alpha_{k} \\
& +2 \sum_{i, j, k} T^{\nabla^{E}}\left(X_{i}, X_{j}, r_{k}\right) \alpha_{i} \wedge \alpha_{j} \wedge \tilde{r}_{k}+C
\end{aligned}
$$


where we used that $T^{\nabla^{E}}\left(\alpha_{i}, \cdot, \cdot\right)=0$ and $T^{\nabla^{E}}\left(r_{i}, r_{j}, X_{k}\right)=0$ from relation (64). Again from relation (64),

$\sum_{i, j, k} T^{\nabla^{E}}\left(X_{i}, X_{j}, X_{k}\right) \alpha_{i} \wedge \alpha_{j} \wedge \alpha_{k}=-\sum_{i, j, k} \mathcal{H}\left(X_{i}, X_{j}, X_{k}\right) \alpha_{i} \wedge \alpha_{j} \wedge \alpha_{k}=-6 \mathcal{H}$

and

$$
T^{\nabla^{E}}\left(X_{i}, X_{j}, r_{k}\right) \alpha_{i} \wedge \alpha_{j} \wedge \tilde{r}_{k}=-\left(R\left(X_{i}, X_{j}\right), r_{k}\right)^{\mathcal{G}} \alpha_{i} \wedge \alpha_{j} \wedge \tilde{r}_{k}
$$

We have proven that $T^{\nabla^{E}}$, as a section of $\Lambda^{3} E$, is given by

$$
T^{\nabla^{E}}=-8 \mathcal{H}-2 \sum_{i, j, k}\left(R\left(X_{i}, X_{j}\right), r_{k}\right)^{\mathcal{G}} \alpha_{i} \wedge \alpha_{j} \wedge \tilde{r}_{k}+C .
$$

This implies

$$
\begin{aligned}
& T^{\nabla^{E}}(\omega \otimes \tau \otimes s)=-8(\mathcal{H} \wedge \omega) \otimes \tau \otimes s+(-1)^{|\omega|} \omega \otimes \tau \otimes C s \\
& +2(-1)^{|\omega|+1} \sum_{i, j, k}\left(R\left(X_{i}, X_{j}\right), r_{k}\right)^{\mathcal{G}}\left(\alpha_{i} \wedge \alpha_{j} \wedge \omega\right) \otimes \tau \otimes \tilde{r}_{k} s .
\end{aligned}
$$

We conclude by combining (80) with (81).

Example 62. Consider a regular Courant algebroid $E$ with surjective anchor $\pi: E \rightarrow T M$ such that $\operatorname{ker} \pi=(\operatorname{ker} \pi)^{\perp}$. A dissection of $E$ defines an isomorphism between $E$ and the Courant algebroid $T M \oplus T M^{*}$ from Example 3 (see Lemma 2.1 of [1]). From Theorem 61, the canonical Dirac generating operator acts on $\Gamma\left(\Lambda T^{*} M\right)$ by $\not(\omega)=d \omega-\mathcal{H} \wedge \omega$. We recover the expression of the Dirac generating operator for exact Courant algebroids, see e.g. [16].

\section{Part III}

\section{Generalized almost Hermitian structures: integrability and spinors}

In Theorem 6.4 of [1] an integrability criterion for a generalized almost complex structure $\mathcal{J}$ on a regular Courant algebroid $E$ with scalar product of neutral signature $(n, n)$, using the canonical Dirac generating operator $\not d: \Gamma(\mathbb{S}) \rightarrow \Gamma(\mathbb{S})$ of $E$ and the pure spinor associated to $\mathcal{J}$, was developed. For completeness of our exposition we recall it in the appendix. As 
an application of the theory from the previous sections, we now characterize the integrability of a generalized almost Hermitian structure $(G, \mathcal{J})$ on $E$ in terms of suitably chosen Dirac operators and the pure spinors associated to $\left.\mathcal{J}\right|_{E_{ \pm}}$, where $E=E_{+} \oplus E_{-}$is the decomposition of $E$ determined by $G$. Remark that rank $E_{+}$and rank $E_{-}$are even (as $\mathcal{J}$ preserves $E_{+}$and $E_{-}$). We consider $E_{ \pm}$endowed with the (non-degenerate) scalar products $\left.\langle\cdot, \cdot\rangle\right|_{E_{ \pm}}$ and we denote by $\mathrm{Cl}\left(E_{ \pm}\right)$the bundle of Clifford algebras over $\left(E_{ \pm},\left.\langle\cdot, \cdot\rangle\right|_{E_{ \pm}}\right)$. We assume that $E_{ \pm}$are oriented and that $\omega_{ \pm}^{2}=1$, where $\omega_{ \pm}$are volume forms determined by positive oriented bases of $E_{ \pm}$, viewed as elements of $\mathrm{Cl}\left(E_{ \pm}\right)$. We assume that there are given irreducible $\mathrm{Cl}\left(E_{ \pm}\right)$-bundles $S_{ \pm}$with Schur algebra $\mathbb{R}$. The latter condition means that any vector bundle morphism $f_{ \pm}: S_{ \pm} \rightarrow S_{ \pm}$which commutes with the $\mathrm{Cl}\left(E_{ \pm}\right)$-action is a multiple of the identity. A quick inspection of Table 1 from [21] (see page 29) implies that either $\left.\langle\cdot, \cdot\rangle\right|_{E_{ \pm}}$have both neutral signature, or $\operatorname{dim} E_{+}=\operatorname{dim} E_{-}$is a multiple of eight, and one of $\left.\langle\cdot, \cdot\rangle\right|_{E_{ \pm}}$is positive definite (while the other is negative definite). Moreover, $S_{ \pm}$are $\mathbb{Z}_{2}$-graded with gradation defined by $S_{ \pm}^{0}:=\frac{1}{2} \gamma_{\left(1+\omega_{ \pm}\right)} S_{ \pm}$and $S_{ \pm}^{1}:=\frac{1}{2} \gamma_{\left(1-\omega_{ \pm}\right)} S_{ \pm}$.

Since $S_{ \pm}$are irreducible $\mathbb{Z}_{2}$-graded $\mathrm{Cl}\left(E_{ \pm}\right)$-bundles, $S_{+} \hat{\otimes} S_{-}$is an irreducible $\mathbb{Z}_{2}$-graded $\mathrm{Cl}(E)$-bundle, with Clifford action given by

$$
\gamma_{v}\left(s_{+} \otimes s_{-}\right)=\gamma_{v_{+}}\left(s_{+}\right) \otimes s_{-}+(-1)^{\left|s_{+}\right|} s_{+} \otimes \gamma_{v_{-}}\left(s_{-}\right),
$$

for any $v=v_{+}+v_{-} \in E$ (see appendix for more details). Remark that $\mathcal{S}=\mathcal{S}_{+} \hat{\otimes} \mathcal{S}_{-}$is the canonical spinor bundle of $S_{+} \hat{\otimes} S_{-}$, where $\mathcal{S}_{ \pm}:=S_{ \pm} \otimes$ $\left|\operatorname{det} S_{ \pm}^{*}\right|^{\frac{1}{r_{ \pm}}}$is the canonical spinor bundle of $S_{ \pm}\left(\right.$and $r_{S_{ \pm}}$is the rank of $S_{ \pm}$).

Lemma 63. If $\eta_{ \pm} \in \Gamma\left(\left(\mathcal{S}_{ \pm}\right)_{\mathbb{C}}\right)$ are pure spinors associated to $\left.\mathcal{J}\right|_{E_{ \pm}}$, then $\eta_{+} \otimes \eta_{-} \in \Gamma\left(\mathcal{S}_{\mathbb{C}}\right)$ is a pure spinor associated to $\mathcal{J}$.

Proof. Let $L$ be the $(1,0)$-bundle of $\mathcal{J}$ and

$$
\left(E_{ \pm}\right)_{\mathbb{C}} \cap L=L_{\eta_{ \pm}}:=\left\{v \in\left(E_{ \pm}\right)_{\mathbb{C}} \mid \gamma_{v} \eta_{ \pm}=0\right\} .
$$

Both $L_{\eta_{+}} \oplus L_{\eta_{-}}$and $L_{\eta_{+} \otimes \eta_{-}}$are subbundles of $\left(E_{+}\right)_{\mathbb{C}} \oplus\left(E_{-}\right)_{\mathbb{C}}=E_{\mathbb{C}}$. From (82), $L_{\eta_{+}} \oplus L_{\eta_{-}} \subset L_{\eta_{+} \otimes \eta_{-}}$. Since $L_{\eta_{+} \otimes \eta_{-}}$is an isotropic subbundle of $E_{\mathbb{C}}$, its rank is at most $n$. By comparing ranks we obtain $L_{\eta_{+}} \oplus L_{\eta_{-}}=L_{\eta_{+} \otimes \eta_{-}}$. As $L=L_{\eta_{+}} \oplus L_{\eta_{-}}$we obtain $L=L_{\eta_{+} \otimes \eta_{-}}$as needed.

Definition 64. The pure spinors $\eta_{ \pm}$from the above lemma are called pure spinors associated to $(G, \mathcal{J})$.

Let $D$ be a generalized Levi-Civita connection of $G$ and $D^{ \pm}$the $E$ connections on $E_{ \pm}$induced by $D$. Choose $E$-connections $D^{S_{ \pm}}$on $S_{ \pm}$compatible with $D^{ \pm}$. Like in the proof of Theorem 41 one can show that $D^{S_{ \pm}}$exist 
and preserve the grading of $S_{ \pm}$, i.e. $D_{e}^{S_{ \pm}} \Gamma\left(S_{ \pm}^{0}\right) \subset \Gamma\left(S_{ \pm}^{0}\right)$ and $D_{e}^{S_{ \pm}} \Gamma\left(S_{ \pm}^{1}\right) \subset$ $\Gamma\left(S_{ \pm}^{1}\right)$, for any $e \in \Gamma(E)$. Since $S_{ \pm}$have Schur algebra $\mathbb{R}$, any other $E$ connection compatible with $D^{ \pm}$is related to $D^{S_{ \pm}}$by $\tilde{D}^{S_{ \pm}}=D^{S_{ \pm}}+\lambda^{ \pm} \otimes \operatorname{Id}_{S_{ \pm}}$, for $\lambda^{ \pm} \in \Gamma\left(E^{*}\right)$. We denote by $D^{\mathcal{S}_{ \pm}}$the $E$-connections induced by $D^{S_{ \pm}}$on $\mathcal{S}_{ \pm}$. Let $D^{\mathcal{S}}:=D^{\mathcal{S}_{+}} \otimes D^{\mathcal{S}_{-}}$be their tensor product (independent of gradations), which is an $E$-connection on $\mathcal{S}=\mathcal{S}_{+} \hat{\otimes} \mathcal{S}_{-}$induced by the $E$-connection $D^{S_{+}} \otimes D^{S_{-}}$on $S_{+} \hat{\otimes} S_{-}$. Recall that we use the convention $v_{ \pm} S_{ \pm}$for the Clifford action $\gamma_{v_{ \pm}} s_{ \pm}$. Similarly, to simplify notation, we write $v\left(s_{+} \otimes s_{-}\right)$instead of $\gamma_{v}\left(s_{+} \otimes s_{-}\right)$, for any $v \in E$.

Lemma 65. The E-connection $D^{\mathcal{S}}$ is compatible with the Clifford action of $\mathrm{Cl}(E)$ on $\mathcal{S}$.

Proof. Using (82) and that $D^{\mathcal{S}_{+}}$preserves the grading of $\mathcal{S}_{+}$, we obtain

$$
D_{e}^{\mathcal{S}}\left(v\left(s_{+} \otimes s_{-}\right)\right)=D_{e}(v)\left(s_{+} \otimes s_{-}\right)+v D_{e}^{\mathcal{S}}\left(s_{+} \otimes s_{-}\right),
$$

for any $e, v \in \Gamma(E)$ and $s_{ \pm} \in \Gamma\left(\mathcal{S}_{ \pm}\right)$, as needed.

In the above setting, there are three Dirac operators to be considered: two Dirac operators $\not D^{\mathcal{S}_{ \pm}}: \Gamma\left(\mathcal{S}_{ \pm}\right) \rightarrow \Gamma\left(\mathcal{S}_{ \pm}\right)$computed using the $E$-connections $D^{\mathcal{S}_{ \pm}}$of the $\mathrm{Cl}\left(E_{ \pm}\right)$-bundles $\mathcal{S}_{ \pm}$, defined by

$$
\not D^{\mathcal{S}_{ \pm}}\left(s_{ \pm}\right):=\frac{1}{2} \sum_{i} \tilde{e}_{i}^{ \pm} D_{e_{i}^{ \pm}}^{\mathcal{S}_{ \pm}} s_{ \pm}
$$

where $\left(e_{i}^{ \pm}\right)$is a basis of $E_{ \pm}$and $\left(\tilde{e}_{i}^{ \pm}\right)$is the metric dual basis, i.e. $\tilde{e}_{i}^{ \pm}$belong to $E_{ \pm}$and $\left\langle e_{i}^{ \pm}, \tilde{e}_{j}^{ \pm}\right\rangle=\delta_{i j}$. The third Dirac operator $\not D^{\mathcal{S}}$ is the one from Section 8, computed using the $E$-connection $D^{\mathcal{S}}$ on the $\mathrm{Cl}(E)$-bundle $\mathcal{S}$. Using that $\left(e_{i}^{+}, e_{j}^{-}\right)$and $\left(\tilde{e}_{i}^{+}, \tilde{e}_{j}^{-}\right)$are bases of $E$ dual with respect to $\langle\cdot, \cdot\rangle$, we obtain that

$$
\not D^{\mathcal{S}}\left(s_{+} \otimes s_{-}\right)=\frac{1}{2} \sum_{i} \tilde{e}_{i}^{+} D_{e_{i}^{+}}^{\mathcal{S}}\left(s_{+} \otimes s_{-}\right)+\frac{1}{2} \sum_{i} \tilde{e}_{i}^{-} D_{e_{i}^{-}}^{\mathcal{S}}\left(s_{+} \otimes s_{-}\right),
$$

for any $s_{ \pm} \in \Gamma\left(\mathcal{S}_{ \pm}\right)$. The next lemma can be checked from definitions.

Lemma 66. The operators $\not D^{\mathcal{S}}, \not D^{\mathcal{S}_{+}}$and $\not D^{\mathcal{S}_{-}}$are related by

$$
\begin{aligned}
& \not \mathcal{S}^{\mathcal{S}}\left(s_{+} \otimes s_{-}\right)=\left(\not D^{\mathcal{S}_{+}} s_{+}\right) \otimes s_{-}+(-1)^{\left|s_{+}\right|} s_{+} \otimes\left(\not D^{\mathcal{S}_{-}} s_{-}\right) \\
& +\frac{1}{2} \sum_{i}\left(\tilde{e}_{i}^{+} s_{+} \otimes\left(D_{e_{i}^{+}}^{\mathcal{S}_{-}} s_{-}\right)+(-1)^{\left|s_{+}\right|}\left(D_{e_{i}^{-}}^{\mathcal{S}_{+}} s_{+}\right) \otimes \tilde{e}_{i}^{-} s_{-}\right),
\end{aligned}
$$

where $s_{ \pm} \in \Gamma\left(\mathcal{S}_{ \pm}\right)$. 
In the next theorem we use the notation $D_{e_{\mp}}^{\mathcal{S}_{ \pm}}\left(\eta_{ \pm}\right) \equiv \eta_{ \pm}$(for $e_{\mp} \in E_{\mp}$ ) if $D_{e_{\mp}}^{\mathcal{S}_{ \pm}}\left(\eta_{ \pm}\right)=f \eta_{ \pm}$for a function $f=f\left(e_{\mp}\right)$ which depends on $e_{\mp}$. By $\not D^{\mathcal{S}_{ \pm}} \eta_{ \pm} \equiv \eta_{ \pm}$we mean $\not D^{\mathcal{S}_{ \pm}} \eta_{ \pm} \in \gamma_{\left(E_{ \pm}\right)_{\mathbb{C}}}\left(\eta_{ \pm}\right)$.

Theorem 67. In the above setting, the generalized almost Hermitian structure $(G, \mathcal{J})$ on the regular Courant algebroid $E$ with scalar product of neutral signature is generalized Kähler if and only if there is a Levi-Civita connection $D$ of $G$ such that

$$
\not D^{\mathcal{S}_{ \pm}} \eta_{ \pm} \equiv \eta_{ \pm}, \quad D_{e_{\mp}}^{\mathcal{S}_{ \pm}} \eta_{ \pm} \equiv \eta_{ \pm}
$$

for any $e_{\mp} \in \Gamma\left(E_{\mp}\right)$. Here $E=E_{+} \oplus E_{-}$is the decomposition determined by $G$ and $\eta_{ \pm} \in \Gamma\left(\mathcal{S}_{ \pm}\right)$are pure spinors associated to $(G, \mathcal{J})$.

Proof. Let $D$ be a Levi-Civita connection of $G$. From (59), $\not d=\not D^{\mathbb{S}}+\frac{1}{4} \gamma_{T}=$ $\not D^{\mathbb{S}}$ since $D$ is torsion-free and, using $D^{\mathbb{S}}=D^{\mathcal{S}} \otimes D^{L}$,

$$
\not D^{\mathbb{S}}(s \otimes l)=\not D^{\mathcal{S}}(s) \otimes l+\frac{1}{2} \sum_{i} \tilde{e}_{i} s \otimes D_{e_{i}}^{L} l, \forall s \in \Gamma(\mathcal{S}), \forall l \in \Gamma(L),
$$

where $\left(e_{i}\right)$ is a basis of $E$ and $\left(\tilde{e}_{i}\right)$ the dual basis with respect to $\langle\cdot, \cdot\rangle$. We obtain that a pure spinor $\eta \otimes l$ from $\mathbb{S}=\mathcal{S} \otimes L$ is projectively closed if and only if $\not D^{\mathcal{S}} \eta \in \gamma_{E_{\mathbb{C}}} \eta$.

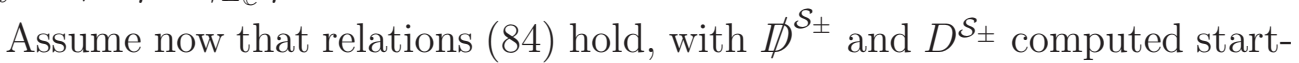
ing with $D$. From (83), we deduce that the pure spinor $\eta=\eta_{+} \otimes \eta_{-}$associated to $\mathcal{J}$ satisfies $\not D^{\mathcal{S}} \eta \in \gamma_{E_{\mathbb{C}}} \eta$, i.e. $\mathcal{J}$ is integrable (see Corollary (72). In a similar way, we show that $\mathcal{J}_{2}=G^{\text {end }} \mathcal{J}$ is integrable. For this, we use the fact $\eta_{+} \otimes \bar{\eta}_{-}$is a pure spinor associated to $\mathcal{J}_{2}$ and $\not D^{\mathcal{S}_{-}} \bar{\eta}_{-} \in \gamma_{\left(E_{-}\right)_{\mathbb{C}}} \bar{\eta}_{-}$(because $\not D^{\mathcal{S}_{-}}: \Gamma\left(\left(\mathcal{S}_{-}\right)_{\mathbb{C}}\right) \rightarrow \Gamma\left(\left(\mathcal{S}_{-}\right)_{\mathbb{C}}\right)$ is the complex linear extension of its restriction to $\mathcal{S}_{-}$and hence commutes with the natural conjugation of $\left.\left(\mathcal{S}_{-}\right)_{\mathbb{C}}\right)$. We obtain that $(G, \mathcal{J})$ is generalized Kähler.

Conversely, assume now that $\mathcal{J}$ is integrable and let $D$ be a Levi-Civita connection of $G$, with $D \mathcal{J}=0$ (which exists from Theorem 36). The relation

$$
D_{e}^{\mathcal{S}_{+}}\left(v \eta_{+}\right)=\left(D_{e}^{+} v\right) \eta_{+}+v D_{e}^{\mathcal{S}_{+}} \eta_{+}, e \in \Gamma(E),
$$

together with the fact that $D$ preserves $L_{\eta_{+}}=L \cap\left(E_{+}\right)_{\mathbb{C}}$ imply that $v D_{e}^{\mathcal{S}_{+}} \eta_{+}=$ 0 , for any $v \in \Gamma\left(L_{\eta_{+}}\right)$, i.e.

$$
D_{e}^{\mathcal{S}_{+}} \eta_{+}=\lambda(e) \eta_{+}, \forall e \in \Gamma(E),
$$

for $\lambda \in \Gamma\left(E^{*}\right)$. Relation (86) with $e:=e_{-} \in \Gamma\left(E_{-}\right)$implies that $D_{e_{-}}^{\mathcal{S}_{+}} \eta_{+} \equiv \eta_{+}$. On the other hand, letting $e:=e_{i}^{+}$in (86), applying the Clifford action of 
$\tilde{e}_{i}^{+}$and summing over $i$ we obtain that $\not D^{\mathcal{S}_{+}} \eta_{+}=\lambda_{+} \eta_{+}$where $\lambda_{+}:=\left.\lambda\right|_{E_{+}}$is a section of $E_{+}^{*} \cong E_{+}$(identified using $\left.\langle\cdot, \cdot\rangle\right|_{E_{+}}$) and $\lambda_{+} \eta_{+}=\gamma_{\lambda_{+}} \eta_{+}$is the Clifford action of $\lambda_{+} \in E_{+}$on $\eta_{+}$. We proved $\not D^{\mathcal{S}_{+}} \eta_{+} \equiv \eta_{+}$as needed. The same argument with $\mathcal{S}_{+}$and $\mathcal{S}_{-}$interchanged shows that all relations (84) are satisfied.

Lemma 68. Relations (84) are independent of the choice of Levi-Civita connection.

Proof. Let $D$ be a Levi-Civita connection. Since $D$ is torsion-free and preserves $E_{ \pm}$, for any $e_{-} \in \Gamma\left(E_{-}\right)$and $v_{+}, w_{+} \in \Gamma\left(E_{+}\right)$,

$$
\begin{aligned}
& 0=T^{D}\left(e_{-}, v_{+}, w_{+}\right)=\left\langle D_{e_{-}} v_{+}-D_{v_{+}} e_{-}-\left[e_{-}, v_{+}\right], w_{+}\right\rangle+\left\langle v_{+}, D_{w_{+}} e_{-}\right\rangle \\
& =\left\langle D_{e_{-}} v_{+}-\left[e_{-}, v_{+}\right], w_{+}\right\rangle
\end{aligned}
$$

which implies $D_{e_{-}} v_{+}=\left[e_{-}, v_{+}\right]_{+}$(see also Lemma 3.2 of [10]). In particular, $D_{e_{-}}^{+}=\left.D_{e_{-}}\right|_{\Gamma\left(E_{+}\right)}$is independent of the choice of Levi-Civita connection $D$, for any $e_{-} \in \Gamma\left(E_{-}\right)$. We obtain that any two $E$-connections $D^{\mathcal{S}_{+}}$and $\tilde{D}^{\mathcal{S}_{+}}$ on $\mathcal{S}_{+}$, compatible with any two Levi-Civita connections of $G$, satisfy $\tilde{D}_{e_{-}}^{\mathcal{S}_{+}}=$ $D_{e_{-}}^{\mathcal{S}_{+}}+\lambda_{-}\left(e_{-}\right) \operatorname{Id}_{\mathcal{S}_{+}}$, for $\lambda_{-} \in E_{-}^{*}$. This implies that the condition $D_{e_{-}}^{\mathcal{S}_{+}} \eta_{+} \equiv \eta_{+}$ is independent of the choice of $D$. In a similar way we prove the statement for $D_{e_{+}}^{\mathcal{S}_{-}} \eta_{-} \equiv \eta_{-}$.

Next, consider two Levi-Civita connections $D$ and $\tilde{D}=D+A$ of $G$. The arguments from Propositions 47 and 49 show that $D^{\mathcal{S}_{+}}$(hence, also $\not D^{\mathcal{S}_{+}}$) depends only on $D^{+}$and

$$
\tilde{\not D}^{\mathcal{S}_{+}}=\not D^{\mathcal{S}_{+}}-\frac{1}{4} \gamma_{\alpha^{+}}-\frac{1}{4} \gamma_{v_{A^{+}}}
$$

where $\alpha^{+} \in \Gamma\left(\Lambda^{3} E_{+}^{*}\right)$ is given by $\alpha^{+}(u, v, w):=\sum_{(u, v, w) \text { cyclic }}\left\langle A_{u} v, w\right\rangle, u, v, w \in$ $E_{+}$and $v_{A^{+}}:=\sum_{i=1}^{n} A_{e_{i}^{+}} \tilde{e}_{i}^{+} \in \Gamma\left(E_{+}\right)$, where $\left(e_{i}^{+}\right)$and $\left(\tilde{e}_{i}^{+}\right)$are $\langle\cdot, \cdot\rangle$-dual bases of $E_{+}$. As $D$ and $\tilde{D}$ are torsion-free, $\alpha^{+}=0$ and we obtain that

$$
\tilde{\not D}^{\mathcal{S}_{+}} \eta_{+}=\not D^{\mathcal{S}_{+}} \eta_{+}-\frac{1}{4} v_{A^{+}} \eta_{+}
$$

This implies that the condition $\not D^{\mathcal{S}_{+}} \eta_{+} \equiv \eta_{+}$is independent of the choice of $D$. In a similar way we prove the statement for $\not D^{\mathcal{S}_{-}} \eta_{-} \equiv \eta_{-}$.

Remark 69. The Dirac operators $\not D^{\mathcal{S}_{ \pm}}$are in fact independent of the LeviCivita connection of $G$, as long as we fix the divergence of $D$. The statement for $\not D^{\mathcal{S}_{+}}$follows from relation (87), by noticing that if $\operatorname{div}_{D}=\operatorname{div}_{\tilde{D}}$ then $v_{A^{+}}=0$ (a similar argument holds for $\not D^{\mathcal{S}_{-}}$). 
From Theorem 67 combined with Lemma 68 we obtain the following characterization for the integrability of generalized almost hyper-Hermitan structures.

Corollary 70. Let $E$ be a regular Courant algebroid with scalar product $\langle\cdot, \cdot\rangle$ of neutral signature. Let $E=E_{+} \oplus E_{-}$be the decomposition of $E$ determined by a generalized metric $G$. Assume that $\left.\langle\cdot, \cdot\rangle\right|_{E_{ \pm}}$are either both of neutral signature, or rank $E_{+}=\operatorname{rank} E_{-}$is a multiple of eight and one of $\left.\langle\cdot, \cdot\rangle\right|_{E_{ \pm}}$ is positive definite (and the other negative definite). A generalized almost hyper-Hermitian structure $\left(G, \mathcal{J}_{1}, \mathcal{J}_{2}, \mathcal{J}_{3}\right)$ is generalized hyper-Kähler if and only if conditions (84) from Theorem 67 hold for a Levi-Civita connection D of $G$ and each of the pure spinors $\eta_{ \pm}^{i}$ associated to $\left(G, \mathcal{J}_{i}\right), i=1,2,3$. The conditions are independent of the choice of $D$.

\section{Appendix}

\section{$11.1 \mathbb{Z}_{2}$-graded algebras and Clifford algebras}

Recall that if $A=A^{0} \oplus A^{1}$ and $B=B^{0} \oplus B^{1}$ are $\mathbb{Z}_{2}$-graded vector spaces, then the tensor product $A \otimes B$ inherits a $\mathbb{Z}_{2}$-gradation

$$
(A \otimes B)^{0}:=A^{0} \otimes B^{0}+A^{1} \otimes B^{1},(A \otimes B)^{1}:=A^{0} \otimes B^{1}+A^{1} \otimes B^{0} .
$$

We denote by $A \hat{\otimes} B$ the vector space $A \otimes B$ together with this gradation. If, moreover, $A$ and $B$ are $\mathbb{Z}_{2}$-graded algebras, then $A \hat{\otimes} B$ inherits the structure of a $\mathbb{Z}_{2}$-graded algebra with multiplication on homogeneous elements defined by

$$
(a \otimes b)(\tilde{a} \otimes \tilde{b}):=(-1)^{|b||\tilde{a}|} a \tilde{a} \otimes b \tilde{b},
$$

where $|a|,|\tilde{b}| \in\{0,1\}$ are the degrees of $a$ and $\tilde{b}$.

We say that a $\mathbb{Z}_{2}$-graded vector space $S=S^{0} \oplus S^{1}$ is a $\mathbb{Z}_{2}$-graded $A$ module if it is a representation space for $A$ and the action of $A$ on $S$ is compatible with gradations, i.e. $A^{i} \cdot S^{j} \subset S^{i+j}$ for any $i, j \in \mathbb{Z}_{2}$.

Finally, if $S$ and $S^{\prime}$ are $\mathbb{Z}_{2}$-graded $A$ - and $B$-modules respectively, then their graded tensor product $S \hat{\otimes} S^{\prime}$ is a $\mathbb{Z}_{2}$-graded $A \hat{\otimes} B$-module with action given by

$$
\gamma_{a \otimes b}\left(s \otimes s^{\prime}\right):=(-1)^{|b||s|} a(s) \otimes b\left(s^{\prime}\right),
$$

where $s \in S, s^{\prime} \in S^{\prime}, a \in A, b \in B,|b|:=\operatorname{deg}(b),|s|:=\operatorname{deg}(s)$ are the degrees of the homogeneous elements $b$ and $s$.

We apply these facts to Clifford algebras and their representations. Assume that $\left(V_{+}, q_{+}\right)$and $\left(V_{-}, q_{-}\right)$are two vector spaces with scalar products 
and let $\left(V:=V_{+} \oplus V_{-}, q:=q_{+}+q_{-}\right)$be their direct sum. As $\mathrm{Cl}\left(V_{ \pm}\right)$are $\mathbb{Z}_{2}$-graded algebras we can consider $\mathrm{Cl}\left(V_{+}\right) \hat{\otimes} \mathrm{Cl}\left(V_{-}\right)$which is a $\mathbb{Z}_{2}$-graded algebra, and as such is isomorphic to $\mathrm{Cl}(V)$ (for the latter statement see e.g. Chapter I of [21]). The isomorphism between $\mathrm{Cl}(V)$ and $\mathrm{Cl}\left(V_{+}\right) \hat{\otimes} \mathrm{Cl}\left(V_{-}\right)$is obtained by extending the map $V \rightarrow \mathrm{Cl}\left(V_{+}\right) \hat{\otimes} \mathrm{Cl}\left(V_{-}\right)$which assigns to any $v=v_{+}+v_{-} \in V_{+} \oplus V_{-}$the vector $v_{+} \otimes 1+1 \otimes v_{2}$.

Let $S_{ \pm}$be $\mathbb{Z}_{2}$-graded $\mathrm{Cl}\left(V_{ \pm}\right)$-modules. From above, the graded tensor product $S_{+} \hat{\otimes} S_{-}$is a $\mathbb{Z}_{2}$-graded $\mathrm{Cl}\left(V_{+}\right) \hat{\otimes} \mathrm{Cl}\left(V_{-}\right)$-module, hence also a $\mathbb{Z}_{2^{-}}$ graded $\mathrm{Cl}(V)$-bundle. Any $v=v_{+}+v_{-} \in V_{+} \oplus V_{-} \subset \mathrm{Cl}(V)$ acts on $S_{+} \hat{\otimes} S_{-}$ as

$$
\gamma_{v_{+}+v_{-}}\left(s_{+} \otimes s_{-}\right)=\gamma_{v_{+}}\left(s_{+}\right) \otimes s_{-}+(-1)^{\left|s_{+}\right|} s_{+} \otimes \gamma_{v_{-}}\left(s_{-}\right) .
$$

\subsection{Integrability of generalized almost complex struc- tures and spinors}

Let $E$ be a regular Courant algebroid with scalar product of signature $(n, n)$, anchor $\pi: E \rightarrow T M$ and canonical Dirac generating operator $\not d: \Gamma(\mathbb{S}) \rightarrow$ $\Gamma(\mathbb{S})$. An almost Dirac structure of $E_{\mathbb{C}}$ is an isotropic complex subbundle of $E_{\mathbb{C}}$ of rank $n$. It is integrable (or a Dirac structure) if is is closed under the (complex linear extension of) the Dorfman bracket of $E$. For a non-vanishing section $\eta \in \Gamma\left(\mathbb{S}_{\mathbb{C}}\right)$ we define

$$
L_{\eta}:=\left\{v \in E_{\mathbb{C}} \mid \gamma_{v} \eta=0\right\} .
$$

The spinor $\eta$ is called pure if $L_{\eta}$ is a vector bundle of rank $n$. Assume that $\eta$ is a pure spinor. A simple computation shows that $L_{\eta}$ is isotropic, and, being of rank $n, L_{\eta}$ is an almost Dirac structure. It is called the null bundle of $\eta$. The assignment $L_{\eta} \rightarrow[\eta]$ is a one-to-one correspondence between almost Dirac structures of $E_{\mathbb{C}}$ and classes of projectively equivalent pure spinors of $\mathbb{S}$ (two pure spinors $\eta_{1}$ and $\eta_{2}$ defined on an open set $U \subset M$ are projectively equivalent if $\eta_{2}=f \eta_{1}$ for a non-vanishing function $f$ on $U$ ). A pure spinor $\eta \in \Gamma\left(\mathbb{S}_{\mathbb{C}}\right)$ is called projectively closed if there is $v \in \Gamma\left(E_{\mathbb{C}}\right)$ such that $\not(\eta)=\gamma_{v} \eta$. (In order to simplify notation, we use the same symbols $\not d$ and $\gamma$ for their complex linear extensions). Note that any pure spinor which is projectively equivalent to a projectively closed spinor is also projectively closed. This follows from $\not(f \eta)=\gamma_{\pi^{*}(d f)}(\eta)+f \not(\eta)$, for any $\eta \in \Gamma\left(S_{\mathbb{C}}\right)$ and $f \in C^{\infty}(M, \mathbb{C})$.

Theorem 71. ([1]) An almost Dirac structure $L$ of $E_{\mathbb{C}}$ is a Dirac structure if and only if, locally, any pure spinor $\eta$ associated to $L$ is projectively closed. 
Proof. Assume that $\eta$ is projectively closed and let $e \in \Gamma\left(E_{\mathbb{C}}\right)$ such that $\not d(\eta)=\gamma_{e} \eta$. Let $v, w \in \Gamma(L)$. Using condition ii) from Definition 39 and $\gamma_{v} \eta=\gamma_{w} \eta=0$, we obtain

$$
\left.\gamma_{[v, w]} \eta=\left[\left[\not d, \gamma_{v}\right], \gamma_{w}\right]\right] \eta=-\gamma_{w} \gamma_{v} \not d(\eta)=-\gamma_{w} \gamma_{v} \gamma_{e} \eta
$$

On the other hand,

$$
\gamma_{w} \gamma_{v} \gamma_{e}=-\gamma_{w} \gamma_{e} \gamma_{v}+2\langle v, e\rangle \gamma_{w}
$$

Combining (89) with (90) and using $\gamma_{v} \eta=\gamma_{w} \eta=0$, we obtain $\gamma_{[v, w]} \eta=0$. This proves that $L$ is a Dirac structure.

Conversely, assume that $L$ is a Dirac structure. Then, for any $v, w \in$ $\Gamma(L),[v, w] \in \Gamma(L)$ and $\gamma_{[v, w]} \eta=0$. This implies, using condition ii) from Definition 39, $\left[\left[d, \gamma_{v}\right], \gamma_{w}\right] \eta=0$, or $\gamma_{w}\left[\phi, \gamma_{v}\right] \eta=0, \forall w \in \Gamma(L)$. We obtain that $\left[\not d, \gamma_{v}\right] \eta$, which is equal to $\gamma_{v} \not(\eta)$, is a multiple of $\eta$, i.e. $\gamma_{v} \not(\eta)=\lambda(v) \eta$ for $\lambda(v) \in C^{\infty}(M, \mathbb{C})$. Remark that $\lambda \in \Gamma\left(L^{*}\right)$. Extend $\lambda$ to a (complex linear) 1 -form on $E_{\mathbb{C}}$ and let $v_{0} \in \Gamma\left(E_{\mathbb{C}}\right)$, such that $2 v_{0}$ is dual to this 1-form with respect to the complex linear extension of $\langle\cdot, \cdot\rangle$. Then

$$
\lambda(v) \eta=2\left\langle v, v_{0}\right\rangle \eta=\gamma_{v} \gamma_{v_{0}} \eta+\gamma_{v_{0}} \gamma_{v} \eta=\gamma_{v} \gamma_{v_{0}} \eta .
$$

The above computations show that $\gamma_{v}\left(\phi(\eta)-\gamma_{v_{0}} \eta\right)=0$, for any $v \in \Gamma(L)$, which implies $\not(\eta)-\gamma_{v_{0}} \eta=g \eta$ for $g \in C^{\infty}(M, \mathbb{C})$. As $\not d$ and $\gamma_{v_{0}}$ are odd operators and pure spinors are chiral, i.e. either even or odd, we conclude $g=0$. This shows that $\not d(\eta)=\gamma_{v_{0}} \eta$, i.e. $\eta$ is projectively closed.

Let $\mathcal{J}$ be a generalized almost complex structure on $E$. The $(1,0)$-bundle $L \subset E_{\mathbb{C}}$ of $\mathcal{J}$ is isotropic with respect to $\langle\cdot, \cdot\rangle$ and satisfies $L \oplus \bar{L}=E_{\mathbb{C}}$. In particular, $\operatorname{rank} L=n$ and $L$ is an almost Dirac structure. A pure spinor $\eta \in \Gamma(\mathbb{S})$ is called associated to $\mathcal{J}$ if $L=L_{\eta}$. From Theorem 71 we obtain:

Corollary 72. A generalized almost complex structure $\mathcal{J}$ on a regular Courant algebroid is integrable if and only if, locally, one (equivalently, any) pure spinor associated to $\mathcal{J}$ is projectively closed.

Acknowledgements. We are grateful to Mario García-Fernández, for explaining to us his characterization of generalized Kähler structures on exact Courant algebroids [12], which we have generalized to regular Courant algebroids in Theorem 67. We are also grateful to Thomas Mohaupt for discussions about Born geometry and for pointing out the reference [9]. We thank Paul Gauduchon for sending us a copy of his paper [14, Carlos Shahbazi for drawing our attention to reference [2], Roberto Rubio for drawing 
our attention to references [13, 24 and Thomas Leistner for useful comments. Research of V.C. was partially funded by the Deutsche Forschungsgemeinschaft (DFG, German Research Foundation) under Germany's Excellence Strategy - EXC 2121 Quantum Universe - 390833306. L.D. was supported by a grant of the Ministry of Research and Innovation, project no PN-IIIID-P4-PCE-2016-0019 within PNCDI.

\section{References}

[1] A. Alekseev, P. Xu, Derived brackets and courant algebroids, unpublished, available at http://www.math.psu.edu/ping/anton-final.pdf (2001).

[2] D. Baraglia, P. Hekmati: Transitive Courant algebroids, String structures and T-duality, Adv. Theor. Math. Physics, vol. 19, (3), (2015), 613-672.

[3] E. Bonan: Sur les G-structures de type quaternionien, Cahiers de Topologie et Géométrie Différentielle, vol. 9, (1967), 389-461.

[4] H. Bursztyn, G. R. Cavalcanti, M. Gualtieri: Reduction of Courant algebroids and generalized complex structures, Adv. Math., vol. 211, (2007), 726-765.

[5] H. Bursztyn, G. Cavalcanti, M. Gualtieri: Generalized Kähler and hyperKähler quotients, Contemporary Mathematics, Poisson Geometry in Mathematics and Physics, vol. 450, 61-77.

[6] G. Cavalcanti, M. Gualtieri: Generalized complex geometry and Tduality, A Celebration of the Mathematical Legacy of Raoul Bott, CRM Proceedings and Lecture Notes, American Mathematical Society (2010), 341-366.

[7] Z. Chen, M. Stiénon, P. Xu: On regular Courant algebroids, Journal of Symplectic Geometry, vol. 11, (1), (2013), 1-24.

[8] A. Coimbra, C. Strickland-Constable, D. Waldram: Supergravity as Generalized Geometry I: Type II Theories, JHEP 11 (2011), 91.

[9] L. Freidel, F. J. Rudolph, D. Svoboda: A Unique Connection for Born Geometry, Comm. Math. Phys. 372, (1), (2019), 119-150. 
[10] M. García-Fernández: Ricci flow, Killing spinors and T-duality in generalized geometry, Adv Math. 350 (2019), 1059-1108.

[11] M. García-Fernández: Torsion-free generalized connections and Heterotic supergravity, Commun. Math. Physics 332 (2014), 89-115.

[12] M. García-Fernández: Private communication, 2018.

[13] M. Garcia-Fernandéz, R. Rubio, C. Tipler: Infinitesimal moduli for the Strominger system and Killing spinors in generalized geometry, Math. Ann., vol. 369, (1-2), (2017), 539-595.

[14] P. Gauduchon: Canonical connections on almost hypercomplex manifolds, Complex analysis and geometry, Eds. V. Ancona, E. Ballico, R.M. Miro Reig, A. Silva, Pitman Research Notes in Mathematics series, 123136.

[15] M. Grützmann, J. P. Michel, P. Xu, Weyl quantization of degree 2 symplectic graded manifolds, arXiv:1410.3346 [math.DG].

[16] M. Gualtieri: Generalized complex geometry, Ann. of Math., vol. 174, (1), (2011), 75-123.

[17] M. Gualtieri: Generalized Kähler Geometry, Comm. Math. Physics, vol. 331, (1), (2014), 297-331.

[18] M. Gualtieri: Branes on Poisson varieties, The Many Facets of Geometry: A Tribute to Nigel Hitchin, Ed. Oscar Garcia-Prada, J. P. Bourguignon, S. Salamon; 368-394, Oxford University Press.

[19] M. Gualtieri: Generalized complex geometry, Ph.D thesis, University of Oxford, 2004.

[20] S. Kobayashi, K. Nomizu: Foundations of Differential Geometry II, Interscience Publishers, 1969.

[21] H. B. Lawson JR., M-L. Michaelson: Spin geometry, Princeton University Press, 1989.

[22] M. Obata: Affine connections in a quaternion manifold and transformations preserving the structure, J. Math. Soc. Japan, vol. 9, (4), (1957), 406-416.

[23] Sternberg: Lectures on Differential Geometry, AMS Chelsea Publishing, vol. 316, 1983. 
[24] K. Uchino: Remarks on the Definition of a Courant algebroid, Letters Math. Physics, vol. 60, (2), (2002), 171-175.

V. Cortés: vicente.cortes@math.uni-hamburg.de

Department of Mathematics and Center for Mathematical Physics, University of Hamburg, Bundesstrasse 55, D-20146, Hamburg, Germany.

L. David: liana.david@imar.ro

Institute of Mathematics Simion Stoilow of the Romanian Academy, Calea Grivitei no. 21, Sector 1, 010702, Bucharest, Romania. 